\title{
On the stability of approximations for the Stokes problem using different finite element spaces for each component of the velocity*
}

\author{
F. Guillén González ${ }^{\dagger}$ and J.R. Rodríguez Galván $¥$
}

December 1, 2014

\begin{abstract}
This paper studies the stability of velocity-pressure mixed approximations of the Stokes problem when different finite element (FE) spaces for each component of the velocity field are considered. We consider some new combinations of continuous FE reducing the number of degrees of freedom in some velocity components. Although the resulting FE combinations are not stable in general, by using the Stenberg's macro-element technique, we show their stability in a wide family of meshes (namely, in uniformly unstructured meshes). Moreover, a post-processing is given in order to convert any mesh family in an uniformly unstructured mesh family. Finally, some $2 D$ and $3 D$ numerical simulations are provided agree with the previous analysis.
\end{abstract}

Keywords. Inf-sup condition, incompressible fluids, mixed variational formulations, finite elements, macro-element technique.

AMS classification (2010). 35Q30, 65N12, 65N30, 76D07.

\section{Contents}

1 Introduction $\quad 2$

2 Definitions and notations. $\mathcal{P}_{0}$ approximation for the pressure 4

3 Stability of the $\left(\mathcal{P}_{1, b}, \mathcal{P}_{1}\right)-\mathcal{P}_{1}$ approximation 5

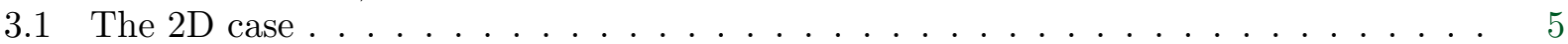

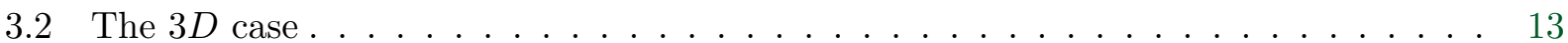

3.3 Numerical simulations . . . . . . . . . . . . . . . . . . . . . 17

4 About stability of $\left(\mathcal{P}_{2}, \mathcal{P}_{1}\right)-\mathcal{P}_{1}$ FE $\quad 23$

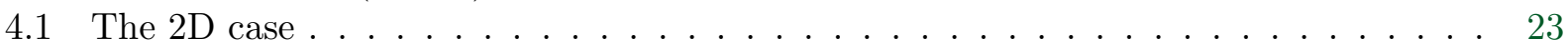

4.2 Numerical simulations . . . . . . . . . . . . . . . . . . . . . . 29

5 Instability of $\left(\mathcal{Q}_{2}, \mathcal{Q}_{1}\right)-\mathcal{Q}_{1}$ in structured meshes

* This work has been partially supported by project MTM2012-32325 (MINECO, Spain). Also the second author has been partially supported by the research group FQM-315 (Junta de Andalucía).

${ }^{\dagger}$ Departamento de Ecuaciones Diferenciales y Análisis Numérico and IMUS. Universidad de Sevilla. Aptdo. 1160, 41080 Sevilla (Spain). guillen@us.es

${ }^{\ddagger}$ Departamento de Matemáticas. Universidad de Cádiz. C.A.S.E.M. Polígono Río San Pedro S/N, 11510 Puerto Real, Cádiz (Spain). rafael.rodriguez@uca.es 


\section{Introduction}

The main purpose of this work is to analyze the stability of velocity-pressure mixed FE approximations of the Stokes problem when the components of velocity are approximated in different spaces.

As described below, this is an issue of great interest for the approximation of some variants of the Navier-Stokes equations (in particular for the approximation of the hydrostatic Navier-Stokes equations governing the large scale ocean [GR14]) and, as far as we know, it has not been sufficiently studied in the literature. In fact, although stability of velocity-pressure mixed FE approximations of the Stokes problem is a matter widely investigated in the last decades, almost all of these works fix the same FE space for all components of the velocity field, being the pressure the only unknown that could be approximated by a different space.

Here we consider the classical Stokes problem in a domain $\Omega \subset \mathbb{R}^{d}(d=2$ or 3$)$,

$$
\begin{aligned}
-\Delta \mathbf{w}+\nabla p=\mathbf{f} & \text { in } \Omega, \\
\nabla \cdot \mathbf{w}=0 & \text { in } \Omega,
\end{aligned}
$$

distinguishing the components of the velocity field as follows: $\mathbf{w}=(\mathbf{u}, v)$, where $\mathbf{u}: \Omega \rightarrow \mathbb{R}^{d-1}$ and $v: \Omega \rightarrow \mathbb{R}$ (horizontal and vertical components, respectively). Also $p: \Omega \rightarrow \mathbb{R}$ is the pressure, f : $\Omega \rightarrow \mathbb{R}^{d}$ is the vector of external forces and, as usual, $\Delta$ and $\nabla \cdot$ represent the Laplace and divergence operators. For sake of simplicity, we take constant viscosity (equal to one) and consider homogeneous Dirichlet boundary condition,

$$
\mathbf{w}=\mathbf{0} \quad \text { on } \partial \Omega .
$$

Let us introduce usual notations: $L^{2}(\Omega)$ represents the space of the square integrable functions in $\Omega$ and $(f, g)=\int_{\Omega} f g$ denotes its scalar product, $H^{m}(\Omega)$ is the space of the functions whose distributional derivatives of order up to $m \in \mathbb{N}$ belong to $L^{2}(\Omega)$ and $H_{0}^{1}(\Omega)$ is the subspace of $H^{1}(\Omega)$ whose functions vanish on $\partial \Omega$.

If one consider the bilinear forms $a(\mathbf{w}, \overline{\mathbf{w}})=(\nabla \mathbf{w}, \nabla \overline{\mathbf{w}})$ and $b(\bar{p}, \mathbf{w})=(\bar{p}, \nabla \cdot \mathbf{w})$, the variational formulation of the Stokes problem (1)-(3) reads: find $(\mathbf{w}, p) \in \mathbf{W} \times P$ such that:

$$
\begin{aligned}
a(\mathbf{w}, \overline{\mathbf{w}})-b(p, \overline{\mathbf{w}}) & =\int_{\Omega} \mathbf{f} \cdot \overline{\mathbf{w}}, \quad \forall \overline{\mathbf{w}} \in \mathbf{W}, \\
b(\bar{p}, \mathbf{w}) & =0, \quad \forall \bar{p} \in P,
\end{aligned}
$$

where $\mathbf{W}=H_{0}^{1}(\Omega)^{d}$ and $P=L_{0}^{2}(\Omega)=\left\{p \in L^{2}(\Omega) / \int_{\Omega} p=0\right\}$. We assume $\mathbf{f}$ in $L^{2}(\Omega)^{d}$.

It is well known (see for instance [BBF13]) that well-posedness of previous problem yields from the coercivity of the bilinear form $a(\cdot, \cdot)$ and the inf-sup condition of $b(\cdot, \cdot)$, which holds for the continuous problem on $\mathbf{W} \times P$. But if we consider the discrete problem, find $\left(\mathbf{w}_{h}, p_{h}\right) \in \mathbf{W}_{h} \times P_{h}$ such that

$$
\begin{aligned}
a\left(\mathbf{w}_{h}, \overline{\mathbf{w}}_{h}\right)-b\left(p_{h}, \overline{\mathbf{w}}_{h}\right) & =\int_{\Omega} \mathbf{f} \cdot \overline{\mathbf{w}}_{h}, \quad \forall \overline{\mathbf{w}}_{h} \in \mathbf{W}_{h}, \\
b\left(\bar{p}_{h}, \mathbf{w}_{h}\right) & =0, \quad \forall \bar{p}_{h} \in P_{h},
\end{aligned}
$$

where $\mathbf{W}_{h} \subset \mathbf{W}$ and $P_{h} \subset \mathbf{W}$ are families of FE spaces, although coercivity of $a(\cdot, \cdot)$ in $\mathbf{W}_{h} \times P_{h}$ is automatically inherited, the continuous inf-sup condition is not enough for well posedness of (4)-(5). 
In fact, each choice of $\mathbf{W}_{h}$ and $P_{h}$ requires checking the following discrete counterpart of the inf-sup condition (also called LBB condition): there exists $\beta>0$ independent on $h$ such that

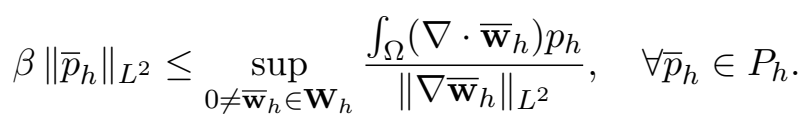

In what follows, we will take $\mathbf{W}_{h}=\mathbf{U}_{h} \times V_{h}$, where $\mathbf{U}_{h}$ and $V_{h}$ are, respectively, FE spaces approximating the (two components of) horizontal velocity and the vertical velocity. If $\Omega \subset \mathbb{R}^{2}, \mathbf{U}_{h}$ is scalar and is denoted by $U_{h}$. If no ambiguity, we denote by $\mathcal{P}_{1}$ or $\mathcal{P}_{2}$ the corresponding continuous piecewise polynomial FE approximation, while $\mathcal{P}_{0}$ denotes discontinuous FE.

The case where $\mathbf{U}_{h}$ and $V_{h}$ are approximated by the same FE space has been widely studied and several combinations (which we denote by $\left(U_{h}, V_{h}\right)-P_{h}$, in the $2 \mathrm{D}$ case) are known satisfying the discrete inf-sup condition (6). That is the case, for example, of $\left(\mathcal{P}_{1, b}, \mathcal{P}_{1, b}\right)-\mathcal{P}_{1}$ (usually known as $\mathcal{P}_{1}+$ bubble or mini-element), $\left(\mathcal{P}_{2}, \mathcal{P}_{2}\right)-\mathcal{P}_{1}$ and $\left(\mathcal{Q}_{2}, \mathcal{Q}_{2}\right)-\mathcal{Q}_{1}$ (Taylor-Hood elements), while some others do not satisfy (6) in general (see for instance Section 2 and references therein). As usual, $\mathcal{P}_{1, b}$ denotes $\mathcal{P}_{1}$ continuous functions enriched by a "bubble" function for each element and $\mathcal{Q}_{k}$ are continuous tensor $\mathcal{P}_{k} \times \mathcal{P}_{k}$ elements on quadrangular meshes.

This work is centered in the case where $\mathbf{U}_{h}$ and $V_{h}$ are approximated by different $\mathrm{FE}$ spaces, for which (as far as we know) only the case $\left(\mathcal{P}_{2}, \mathcal{P}_{1}\right)-\mathcal{P}_{0}$ has been addressed, see [Ste90]. Some new combinations $\left(\mathbf{U}_{h}, V_{h}\right)-P_{h}$ are going to be studied, showing (or refusing) its stability in wide mesh families and extending the analysis to three-dimensional domains.

Specifically, our main contribution is showing that although the inf-sup condition (6) does not hold in general for the combinations $\left(\mathcal{P}_{1, b}, \mathcal{P}_{1}\right)-\mathcal{P}_{1}$ and $\left(\mathcal{P}_{2}, \mathcal{P}_{1}\right)-\mathcal{P}_{1}$ (and also for the symmetric cases $\left(\mathcal{P}_{1}, \mathcal{P}_{1, b}\right)-\mathcal{P}_{1}$ and $\left.\left(\mathcal{P}_{1}, \mathcal{P}_{2}\right)-\mathcal{P}_{1}\right)$, it is satisfied in numerous mesh families, namely in uniformly unstructured meshes (see Definition 7 ). In fact, one can easily detect if a given mesh is not unstructured and, with a slight post-processing, transform it into a uniformly unstructured one (see Algorithm 1 below).

There are some other well-known unstable elements (for instance $\left(\mathcal{P}_{1}, \mathcal{P}_{1}\right)-\mathcal{P}_{0}$ and $\left(\mathcal{P}_{1, b}, \mathcal{P}_{1}\right)-\mathcal{P}_{0}$, see Section 2) which have less degrees of freedom than the "mini-element" $\left(\mathcal{P}_{1, b}, \mathcal{P}_{1, b}\right)-\mathcal{P}_{1}$ and that are stable just in some concrete meshes. These meshes are, in particular, uniformly unstructured and indeed FE combinations provided in this paper are stable in a very much larger family of meshes. In this sense, $\left(\mathcal{P}_{1, b}, \mathcal{P}_{1}\right)-\mathcal{P}_{1}$ constitutes a "minimal-element" because with less degrees of freedom than the "mini-element" stability is provided for unstructured meshes (or slightly post-processed structured ones).

Previous results are extended to $3 D$ domains by using $\left(\mathcal{P}_{1, b}, \mathcal{P}_{1, b}, \mathcal{P}_{1}\right)-\mathcal{P}_{1}$ and even $\left(\mathcal{P}_{1, b}, \mathcal{P}_{1}, \mathcal{P}_{1}\right)-\mathcal{P}_{1}$ (and the corresponding spaces replacing $\mathcal{P}_{1, b}$ by $\mathcal{P}_{2}$ ). Additionally, we present some numerical tests showing that $\left(\mathcal{P}_{1, b}, \mathcal{P}_{1}\right)-\mathcal{P}_{1}$ preserves the accuracy $O(h)$ while $\left(\mathcal{P}_{2}, \mathcal{P}_{1}\right)-\mathcal{P}_{1}$ presents a loss of order (only order $O(h)$ ) with respect to the order $O\left(h^{2}\right)$ of the classical Taylor-Hood approximation $\left(\mathcal{P}_{2}, \mathcal{P}_{2}\right)-\mathcal{P}_{1}$.

Our interest on the stability of anisotropic horizontal/vertical velocity approximation has been motivated by the study of some geophysical fluid models governing the motion of large scale oceans (see for instance [CB09] and references therein cited). In fact, they lead to the Navier-Stokes equations with anisotropic eddy viscosities in shallow domains or, after rescaling, to equivalent models in adimensional domains [AG01, BL92]. In the linear steady case, the following Anisotropic Stokes problem arises:

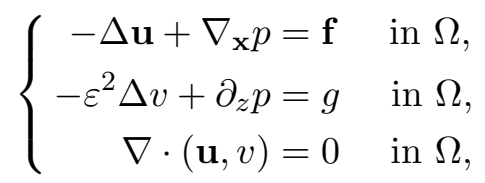


where $\varepsilon>0$ is the so-called aspect ratio between vertical and horizontal scales. When $\varepsilon \rightarrow 0$, diffusion for the vertical velocity vanishes [BL92] and a singular limit problem appears, which is called the Hydrostatic Stokes equations. This type of problems also appear in the transient nonlinear case (called Hydrostatic Navier-Stokes equations or Primitive Equations of the ocean), see [AG01].

In a forthcoming work [GR14] (see also [Azé94, Azé96]) the well-posedness of the approximations in uniformly unstructured meshes of (AS) is developed (valid when $\varepsilon$ is "small" or zero). This theory is based on the Stokes inf-sup condition jointly to an additional inf-sup condition needed to stabilize the vertical velocity. This new inf-sup condition is satisfied at discrete level when the pressure space is large enough with respect to the vertical velocity one. But, for standard Stokes FE spaces, the when the number of degrees of freedom for pressure is too large then Stokes stability is not satisfied. The question is, are there intermediate possibilities for approximating velocity-pressure mixed formulation satisfying both stability constraints? The answer given in this paper is yes, but for uniformly unstructured meshes.

This paper is organized as follows. In Section 2, we recall some results about approximations with discontinuous pressure. In Section 3 we prove that $\left(\mathcal{P}_{1, b}, \mathcal{P}_{1}\right)-\mathcal{P}_{1}$ is stable in uniformly unstructured meshes and show its instability in some structured meshes. Results are generalized to the $3 D$ case and numerical tests are shown confirming our analysis. In Section 4 , we show that $\left(\mathcal{P}_{2}, \mathcal{P}_{1}\right)-\mathcal{P}_{1}$ is also stable in most unstructured meshes, although the results are slightly more restrictive than for $\left(\mathcal{P}_{1, b}, \mathcal{P}_{1}\right)-\mathcal{P}_{1}$ approximation. This theory is supported by some numerical experiments, including some $3 D$ tests. Finally, in Section 5 , we show instability of the combination $\left(\mathcal{Q}_{2}, \mathcal{Q}_{1}\right)-\mathcal{Q}_{1}$ on quadrangular elements, both analytical and computationally.

\section{Definitions and notations. $\mathcal{P}_{0}$ approximation for the pressure}

Let us assume elsewhere that $\Omega \subset \mathbb{R}^{d}$, with $d=2$ or $d=3$, is a domain with polygonal boundary and let $\mathcal{T}_{h} \subset \mathbb{R}^{d}$ be a shape regular family of triangulations of $\bar{\Omega}$. Furthermore, let us suppose that $\mathcal{T}_{h}$ is a simplicial mesh (i.e. composed of $2 D$ triangles or $3 D$ tetrahedrons), unless otherwise stated.

In this section we recall some results about discontinuous approximation of pressure. Specifically, the case $d=2$ is considered and, for the approximation of the discrete Stokes problem (4)-(5), the following $\mathcal{P}_{0}$ discontinuous space for the approximation of the pressure is introduced:

$$
P_{h}=\left\{p_{h} \in L_{0}^{2}(\Omega) /\left.p\right|_{T} \in \mathcal{P}_{0} \forall T \in \mathcal{T}_{h}\right\} .
$$

About velocity, we define $\mathbf{W}_{h}=U_{h} \times V_{h}$, where $U_{h}$ and $V_{h}$ are continuous FE spaces for horizontal and vertical velocity, respectively. Indeed, we deal $\mathcal{P}_{1}, \mathcal{P}_{1, b}$ or $\mathcal{P}_{2}$ spaces for horizontal velocity and $\mathcal{P}_{1}$ for vertical one. Of course the symmetric choice is equivalent.

As result, we consider the FE combinations denoted by $\left(\mathcal{P}_{1}, \mathcal{P}_{1}\right)-\mathcal{P}_{0},\left(\mathcal{P}_{1 b}, \mathcal{P}_{1}\right)-\mathcal{P}_{0}$ and $\left(\mathcal{P}_{2}, \mathcal{P}_{1}\right)-\mathcal{P}_{0}$ whose stability has been sufficiently analyzed in literature and we can summarize as follows: $\left(\mathcal{P}_{2}, \mathcal{P}_{1}\right)-\mathcal{P}_{0}$ is the minimal FE combination (with $\mathcal{P}_{0}$ discontinuous pressure) which is stable in general meshes. Namely, let us consider

$$
V_{h}=\left\{v_{h} \in H_{0}^{1}(\Omega) \cap C^{0}(\bar{\Omega}) /\left.v\right|_{T} \in \mathcal{P}_{1}, \forall T \in \mathcal{T}_{h}\right\}
$$

- Instability of $\left(\mathcal{P}_{1}, \mathcal{P}_{1}\right)-\mathcal{P}_{0}$ approximation, i.e. the case where $U_{h}=V_{h}$, is well known to occur excepting some specific meshes (called criscross meshes, see [QZ07]). They are particular cases of $x$-unstructured and $y$-unstructured meshes, see Definition 6.

- Instability of $\left(\mathcal{P}_{1, b}, \mathcal{P}_{1}\right)-\mathcal{P}_{0}$, i.e. the case where

$$
U_{h}=\left\{u_{h} \in H_{0}^{1}(\Omega) \cap C^{0}(\bar{\Omega}) /\left.u_{h}\right|_{T} \in \mathcal{P}_{1, b}(T), \forall T \in \mathcal{T}_{h}\right\}
$$


can be easily reduced to the above case, using the fact that integrals in triangles of derivatives of bubble functions vanish. The case $\left(\mathcal{P}_{1, b}, \mathcal{P}_{1, b}\right)-\mathcal{P}_{0}$ is similar.

- Stability of $\left(\mathcal{P}_{2}, \mathcal{P}_{1}\right)-\mathcal{P}_{0}$, i.e. the case where

$$
U_{h}=\left\{u_{h} \in H_{0}^{1}(\Omega) \cap C^{0}(\bar{\Omega}) /\left.u_{h}\right|_{T} \in \mathcal{P}_{2}, \forall T \in \mathcal{T}_{h}\right\}
$$

while $V_{h}$ and $P_{h}$ are defined respectively in (8) and (7), was shown by R. Stenberg in [Ste90], using the macro-element technique (which was developed by himself in that reference and [Ste84]).

\section{$3 \quad$ Stability of the $\left(\mathcal{P}_{1, b}, \mathcal{P}_{1}\right)-\mathcal{P}_{1}$ approximation}

In what follows, continuous piecewise linear pressures are selected:

$$
P_{h}=\left\{p_{h} \in L_{0}^{2}(\Omega) \cap C^{0}(\bar{\Omega}) /\left.p\right|_{T} \in \mathcal{P}_{1}, \forall T \in \mathcal{T}_{h}\right\} .
$$

Using the macro-element technique, we study the $2 \mathrm{D}$ case, where $U_{h}$ is a continuous $\mathcal{P}_{1, b} \mathrm{FE}$ space, as defined in (9) and $V_{h}$ is a continuous $\mathcal{P}_{1}$ space, as in (8). Results are generalized to the $3 D$ case (Section 3.2) and some numerical experiments are shown, supporting these results (Section 3.3).

\subsection{The $2 \mathrm{D}$ case}

For the study of the $\left(\mathcal{P}_{1, b}, \mathcal{P}_{1}\right)-\mathcal{P}_{1}$ approximation, the macro-element technique is applied, following the seminal papers of Stenberg [Ste84, Ste90] (specially the former). We show a local stability result (Theorem 3) and global stability result (Theorem 4), where a generalization of Stenberg's theory must be introduced.

A macro-element partitioning, $\mathcal{M}_{h}$, of a triangulation $\mathcal{T}_{h}$ in $\Omega$ is a family of connected sets $M \in \mathcal{M}_{h}$ which are union of at least two elements of $\mathcal{T}_{h}$. For each $M \in \mathcal{M}_{h}$, let $U_{0, M}$ and $V_{0, M}$ be the spaces of functions in $H_{0}^{1}(M) \cap C^{0}(\bar{M})$ which are, respectively, in $\mathcal{P}_{1, b}$ and $\mathcal{P}_{1}$ for all $T \subset M$. Let $P_{M}$ be the space of functions in $C^{0}(\bar{M})$ which are in $\mathcal{P}_{1}$ for every $T \subset M$.

Definition 1. We say that $\left(U_{h}, V_{h}\right)-P_{h}$ is regular in a macro-element $M$ if

$$
\text { The set } N_{M}=\left\{p_{h} \in P_{M}, \int_{M}\left(\partial_{x} u_{h}+\partial_{y} v_{h}\right) p_{h}=0 \forall\left(u_{h}, v_{h}\right) \in U_{0, M} \times V_{0, M}\right\}
$$

only consists of the functions which are constant on $M$.

In other case, $\left(U_{h}, V_{h}\right)-P_{h}$ is said singular in $M$.

Stenberg shown, roughly speaking, that if this regularity condition is satisfied, independently of the geometrical shape of the macro-elements of some given partitioning (and under slight topological restrictions on macro-elements, formulated in Theorem 3 below) the global inf-sup condition (6) holds uniformly (i.e. with a constant $\beta$ independent of the macro-element).

More specifically, according to the theory of Stenberg, we introduce the following definition:

Definition 2. A macro-element $M$ is said to be equivalent (in the sense of Stenberg) to a reference macro-element $M^{*}$ if there exists a continuous one-to-one application $F_{M}: M^{*} \rightarrow M$ which consistently maps elements contained in $M^{*}$ to elements in $M$ (see [Ste84] or [Ste90] for details).

The following result holds (see Theorem 3.1 in [Ste90]): 
Theorem 1. Suppose that there exists a family of macro-elements, $\mathcal{M}_{h}$, of $\mathcal{T}_{h}$ which is composed of a fixed set of equivalence (in the sense of Stenberg) classes $\mathcal{E}_{n}$ of macro-elements, $n=1, \ldots, N$ and a positive integer $L$ ( $N$ and $L$ independent of $h$ ) such that:

(M1) The condition (12) is satisfied for every $M \in \mathcal{M}_{h}$ (i.e. $\left(U_{h}, V_{h}\right)-P_{h}$ is regular in macroelements).

(M2) Each $M \in \mathcal{M}_{h}$ belongs to one of the classes $\mathcal{E}_{n}, n=1, \ldots, N$.

(M3) If $e$ is an edge (or face) of an element of $\mathcal{T}_{h}$ which is interior to $\Omega$, then $e$ is interior to at least one and no more than $L$ macro-elements of $\mathcal{M}_{h}$.

Then the Stokes discrete inf-sup condition (6) holds for $\left(U_{h}, V_{h}\right)-P_{h}$.

In this work we focus on a concrete set of families of macro-elements, which are defined as union of the elements of $\mathcal{T}_{h}$ which share one only interior vertex. More precisely, let us denote by $\mathcal{T}_{h}$ the set of all of the vertices of $\mathcal{T}_{h}$ which are in the interior of $\Omega$.

\section{Definition 3.}

- We say that a macro-element $\widehat{M}$ is centered in a vertex $q \in \stackrel{\circ}{\mathcal{T}}_{h}$ if $\widehat{M}$ is the union of every $T \in \mathcal{T}_{h}$ such that $q$ is a vertex of $T$.

- We define a vertex-centered macro-element partitioning of $\mathcal{T}_{h}$, denoted by $\widehat{\mathcal{M}}_{h}$, as any macroelement partitioning of $\mathcal{T}_{h}$ such that every $\widehat{M} \in \widehat{\mathcal{M}}_{h}$ is centered in some vertex $q \in \mathcal{T}_{h}$.

If $\widehat{M}$ is centered in $q_{0}$, we denote by $\mathrm{n}_{v}^{q_{0}}$ (or just $\mathrm{n}_{v}$ ) the number of vertices $q$ in $\widehat{M}$ such that $q \neq q_{0}$. See that $\mathrm{n}_{v}$ matches also the number of elements contained in $\widehat{M}$. For example, Figure 1 shows two macro-elements, each of which is centered in a vertex, $q_{0}$, with $\mathrm{n}_{v}=5$ in both cases.

Shape regularity of $\mathcal{T}_{h}$ implies that $\exists N \in \mathbb{N}$ (independent of $h$ ) such that each vertex of $\mathcal{T}_{h}$ is in no more than $N$ elements. On the other hand, if $\mathcal{T}_{h}$ is a simplicial mesh family in $\mathbb{R}^{d}$, one has $d<\mathrm{n}_{v}^{q}$. Therefore:

$$
d<\mathrm{n}_{v}^{q} \leq N, \quad \forall q \in \stackrel{\circ}{\mathcal{T}}_{h}
$$

Fixed a reference macro-element $\widehat{M}^{*}$ (centered in a vertex $q^{*} \in \stackrel{\circ}{\mathcal{T}}_{h}$ ) and fixed $n=\mathrm{n}_{v}^{q^{*}}$, we denote by $\widehat{\mathcal{E}}_{n}$ the family of macro-elements which are equivalent (in the sense of Stenberg) to $\widehat{M}^{*}$. Thus a macro-element partitioning $\widehat{\mathcal{M}}_{h}$ is whatever such that every $M \in \widehat{\mathcal{M}}_{h}$ belongs to some $\widehat{\mathcal{E}}_{n}$, with $n \in \mathbb{N}$. Hereafter we assume that $\mathcal{T}_{h}$ satisfies the following slightly restrictive hypothesis:

Assumption 1. Each element $T \in \mathcal{T}_{h}$ has at least one vertex in the interior of, $\Omega$.

According to (13), for any simplex mesh system $\mathcal{T}_{h}$, one can build at least one and at most $N-d$ families of vertex-centered macro-elements, $\widehat{\mathcal{E}}_{d+1}, \widehat{\mathcal{E}}_{d+2} \ldots, \widehat{\mathcal{E}}_{N}$. Assumption 1 ensures that $\mathcal{T}_{h}$ can be covered by macro elements of these families.

Lemma 2. Any vertex-centered macro-element partitioning $\widehat{\mathcal{M}}_{h}$ satisfies (M2) and (M3).

Proof. Only (M3) must be satisfied. If $e$ is an edge (or face) in the interior of $\Omega$, then it is adjacent to, at least, one interior vertex, $q_{0}$ and then $e$ is interior to, at least, the macro-element centered in $q_{0}$. On the other hand $e$ is interior to (at most) $d$ macro-elements: that one which are centered in $q_{0}$ and those ones centered in $q_{i}, i=1, \ldots, d-1$, where $q_{i}$ are the other vertices of $e$. 


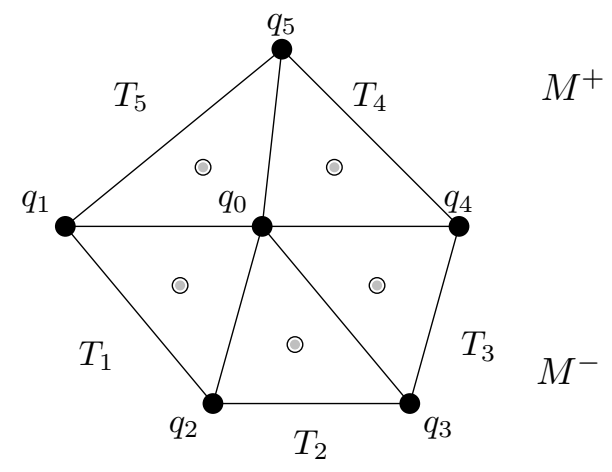

(a) Macroelement that can be split by an horizontal line (then, it is $y$-structured and $\left(\mathcal{P}_{1, b}, \mathcal{P}_{1}\right)-\mathcal{P}_{1}$ is not regular in it).

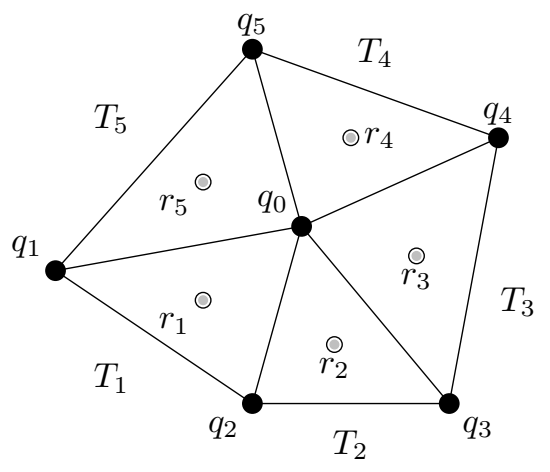

(b) Macroelement that cannot be split by any straight line (it is $x$-unstructured and $y$-unstructured).

Figure 1: Bubble macroelements.

Thus (in meshes satisfying Assumption 1) Lemma 2 and Theorem 1 state that accomplishing the regularity condition (M1) in some vertex-centered macro-element partitioning $\widehat{\mathcal{M}}_{h}$ is sufficient for inf-sup condition (6). The drawback is that, as it is shown below, not all of vertex-centered macroelements satisfy hypothesis (M1). Next we provide a result characterizing the macro-elements of $\widehat{\mathcal{M}}_{h}$ where that hypothesis (in fact (12)) holds for $\left(\mathcal{P}_{1, b}, \mathcal{P}_{1}\right)-\mathcal{P}_{1}$. The idea is checking the macro-element structure in the following sense.

Definition 4. Let $\mathcal{T}_{h}$ be a triangulation of $\Omega \subset \mathbb{R}^{d}$ (in practice, $d=2$ or 3 ) and let $M$ be a macro-element in $\mathcal{T}_{h}$. We say that $M$ can be split by an hyperplane $\Pi$ if there exists two other macro-elements $M_{1}$ and $M_{2}$, composed of elements of $\mathcal{T}_{h}$, such that $M_{1} \cup M_{2}=M$ and $M_{1} \cap M_{2} \subset \Pi$.

Definition 5. A macro-element $M$ of a mesh $\mathcal{T}_{h}$ is be said $x$-structured if $M$ can be split by an hyperplane $x=C$, for some $C \in \mathbb{R}$. In other case, it is said $x$-unstructured.

Note that a $x$-structured macro-element can be split by a straight line (a plane, in the $3 D$ case) which is orthogonal to the $x$ axis. Similarly, a whole mesh $\mathcal{T}_{h}$ is said $x$-structured if it can be split by an hyperplane $x=C, C \in \mathbb{R}$. Analogue definitions can be provided for $y$-structured and $z$-structured macro-elements or meshes.

For example:

- Figure 1a represents a $2 D$ macro-element that can be split by an horizontal line (and not by a vertical line), then it is $y$-structured (and $x$-unstructured).

- The whole mesh in Figure 3 (i.e. all its vertex-centered macro-elements) is $x$-structured and $y$-structured.

- The macro-element represented in Figure $1 \mathrm{~b}$ cannot be split by any horizontal or vertical straight line, therefore it is $x$-unstructured and $y$-unstructured.

The following result relates the regularity in a macro-element with its structure. In fact, it states that, in those macro-elements which are unstructured in some direction, the bubble functions can be safely removed from the corresponding component of the velocity field.

Theorem 3. Let $\widehat{M}$ be a macro-element in a vertex-centered partitioning $\widehat{\mathcal{M}}_{h}$. Then: 
1. The combination $\left(\mathcal{P}_{1, b}, \mathcal{P}_{1}\right)-\mathcal{P}_{1}$ is regular in $\widehat{M}$ (namely (12) holds for $\widehat{M}$ ) if and only if $\widehat{M}$ is $y$-unstructured.

2. The combination $\left(\mathcal{P}_{1}, \mathcal{P}_{1, b}\right)-\mathcal{P}_{1}$ is regular in $\widehat{M}$ if and only if $\widehat{M}$ is $x$-unstructured.

This Theorem can also be interpreted as follows: the standard mini-element $\left(\mathcal{P}_{1, b}, \mathcal{P}_{1, b}\right)-\mathcal{P}_{1}$ is "too rich" in those macro-elements which are unstructured in the $x$ or $y$ direction.

Proof. We show only the first statement, because the second one is symmetric.

$\Leftarrow$ Let $\widehat{M} \in \widehat{\mathcal{M}}_{h}$ be a macro-element with $q_{0}$ as unique interior vertex. Let $\mathrm{n}_{v}=\mathrm{n}_{v}^{q_{0}}, q_{i}\left(i=1, \ldots \mathrm{n}_{v}\right)$ the number of elements around $q_{0}$ and $r_{i}\left(i=1, \ldots, \mathrm{n}_{v}\right)$ the bubble degrees of freedom (barycentres of the triangles in $\widehat{M}$ ). If $\widehat{M}$ is $y$-unstructured then there are not two vertices $q_{i}$ and $q_{j}$ in $\widehat{M}$ horizontally aligned to $q_{0}$. For each $p_{h} \in N_{\widehat{M}}$, that is $\int_{\widehat{M}}\left(\partial_{x} u_{h}+\partial_{y} v_{h}\right) p_{h}=0$, let us denote the shape functions

$$
\left.p_{h}\right|_{T_{i}}=a_{i}+b_{i} x+c_{i} y, \quad \forall T_{i} \in \widehat{M} .
$$

First let us choose $v_{h}=0$ and $u_{h} \in U_{0, \widehat{M}}$ such that $u_{h}=1$ on a barycentre $r_{i}$ and $u_{h}=0$ on all other degrees of freedom in $\widehat{M}\left(r_{j}, j=1, \ldots, \mathrm{n}_{v}, j \neq i\right.$ and $\left.q_{k}, k=0, \ldots, \mathrm{n}_{v}\right)$, that is $u_{h}$ is a bubble function on $T_{i}$. Then,

$$
0=\int_{\widehat{M}}\left(\partial_{x} u_{h}+\partial_{y} v_{h}\right) p_{h}=\int_{T_{i}} \partial_{x} u_{h} p_{h}=-\int_{T_{i}} u_{h} \partial_{x} p_{h}=-b_{i} \int_{T_{i}} u_{h},
$$

hence

$$
b_{i}=0 \quad \forall i=1, \ldots, \mathrm{n}_{v},
$$

in particular $\partial_{x} p_{h}=0$ in $\widehat{M}$. Now, imposing the continuity of $p_{h}$ on $q_{0}$ and assuming, without loss of generality, $q_{0}=(0,0)$, it is straightforward that there exists $a \in \mathbb{R}$ such that

$$
a_{1}=\ldots=a_{\mathrm{n}_{v}}=a .
$$

On the other hand, imposing the continuity of $p_{h}$ in the remaining vertices $q_{i}=\left(q_{i}^{x}, q_{i}^{y}\right) \in \partial \widehat{M}$ the common vertex to $T_{i}$ and $T_{i+1}$ (denoting $T_{\mathrm{n}_{v}+1}=T_{1}$ ), one has:

$$
q_{i}^{y}\left(c_{i+1}-c_{i}\right)=0, \quad i=1, \ldots, \mathrm{n}_{v},
$$

where (14) and (15) have been applied. Therefore if $q_{i}^{y} \neq 0$ for all $i$, that is if there is no vertex horizontally aligned with $q_{0}$, then there exists $c \in \mathbb{R}$ such that

$$
c_{1}=\ldots=c_{\mathrm{n}_{v}}=c .
$$

This equality is also true when there is only one vertex horizontally aligned with $q_{0}$, because in (17) there is one equation plus than unknowns $c_{1}, \ldots, c_{\mathrm{n}_{v}}$.

Finally let us choose $u_{h}=0$ and $v_{h} \in V_{0, \widehat{M}}$ defined as $v_{h}=1$ in $q_{0}$ and $v_{h}=0$ in all other degrees of freedom $\left(q_{i}, i=1, \ldots, \mathrm{n}_{v}\right)$. Applying the quadrature formula $\int_{T} f d x d y=\frac{|T|}{3} \sum_{i=1}^{3} f\left(q_{i}^{T}\right)$ with $q_{i}^{T}$ the vertices of $T$, which is exact in $\mathcal{P}_{1}$,

$$
0=\int_{\widehat{M}} \partial_{y} v_{h} p_{h}=-\sum_{i=1}^{\mathrm{n}_{v}} \int_{T_{i}} v_{h} \partial_{y} p_{h}=-\sum_{i=1}^{\mathrm{n}_{v}} c_{i} \frac{\left|T_{i}\right|}{3}
$$

and considering (17) we conclude $c_{i}=0$ for all $i$. Consequently, $p_{h}$ is constant on $\widehat{M}$ and macroelement condition (12) holds. 
Assume there are two other vertices horizontally aligned with $q_{0}=(0,0)$ (without loss of generality they are being indexed $q_{j}$ and $q_{\mathrm{n}_{v}}$ ). From (16) we only deduce:

$$
\begin{aligned}
c_{1} & =c_{2}=\ldots=c_{j}, \\
c_{j+1} & =c_{j+2}=\ldots=c_{\mathrm{n}_{v}},
\end{aligned}
$$

and in this case, for some $c, \widetilde{c} \in \mathbb{R}$,

$$
\partial_{y} p_{h}(x, y)= \begin{cases}c & \text { if } y>0 \\ \widetilde{c} & \text { if } y \leq 0\end{cases}
$$

We are going to use this idea to obtain a counterexample. Indeed, now $\widehat{M}$ can be split into two disjoint regions: $\widehat{M}^{+}=\{(x, y) \in \widehat{M} / y>0\}$ and $\widehat{M}^{-}=\{(x, y) \in \widehat{M} / y<0\}$, each of which can be written as union of triangles of $\widehat{M}$ (see Figure $1 \mathrm{~b}$ ). For any parameters $a$ and $c \in \mathbb{R}$, let us define $p_{h} \in P_{\widehat{M}}$ as follows:

$$
p_{h}(x, y)= \begin{cases}a-\frac{c}{\left|\widehat{M}^{+}\right|} y & \text { if }(x, y) \in \widehat{M}^{+}, \\ a+\frac{c}{\left|\widehat{M}^{-}\right|} y & \text { if }(x, y) \in \widehat{M}^{-},\end{cases}
$$

where $|R|$ denotes the area of a region $R \subset \mathbb{R}^{2}$. Every $v_{h} \in V_{0, \widehat{M}}$ can be characterized as the $\mathcal{P}_{1}$ basis function that is equal to $\alpha=v_{h}\left(q_{0}\right)$ in the vertex $q_{0}$ and 0 in all other vertices in $\widehat{M}$. Hence one has (applying the above mass-lumping quadrature formula):

$$
\begin{aligned}
\int_{\widehat{M}} \partial_{y} v_{h} p_{h} & =-\int_{\widehat{M}} v_{h} \partial_{y} p_{h}=\frac{c}{\left|\widehat{M}^{+}\right|} \int_{\widehat{M}^{+}} v_{h}-\frac{c}{\left|\widehat{M}^{-}\right|} \int_{\widehat{M}^{-}} v_{h} \\
& =\frac{c}{\left|\widehat{M}^{+}\right|} \sum_{T \subset \widehat{M}^{+}} \frac{|T|}{3} \alpha-\frac{c}{\left|\widehat{M}^{-}\right|} \sum_{T \subset \widehat{M}^{-}} \frac{|T|}{3} \alpha=\frac{c}{3} \alpha-\frac{c}{3} \alpha=0
\end{aligned}
$$

Then, taking into account that $\partial_{x} p_{h}=0$, the equality $\int_{\widehat{M}}\left(\partial_{x} u_{h}+\partial_{y} v_{h}\right) p_{h}=0$ is satisfied for all $\left(u_{h}, v_{h}\right) \in U_{0, \widehat{M}} \times V_{0, \widehat{M}}$, therefore (12) does not hold.

Now, we are interested in extending the local stability result of Theorem 4 to a global result for the whole $\mathcal{T}_{h}$. According to Theorem 1 and Lemma 2, in order to apply the macro-element theory for the particular family $\widehat{\mathcal{M}}_{h}$, it must be shown that macro-element regularity condition (12) holds for all $\widehat{M} \in \widehat{\mathcal{M}}_{h}$, namely for every macro-element in the families $\widehat{\mathcal{E}}_{d+1}, \ldots, \widehat{\mathcal{E}}_{N}$. The drawback is that $y$-structured macro-elements are part of these families, and regularity condition is not satisfied for $y$-structured macro-elements, according to Theorem 4 (in the case of $\left(\mathcal{P}_{1, b}, \mathcal{P}_{1}\right)-\mathcal{P}_{1}$ ).

Note that even if a concrete mesh family, $\mathcal{T}_{h}$, is built where every $\widehat{M}$ is $y$-unstructured (and then regularity condition (12) is satisfied for every $\widehat{M}$ ) one cannot apply Theorem 1 to conclude that inf-sup condition (5) holds. Indeed, in Theorem $1, \mathcal{M}_{h}$ is composed of every of the equivalent macro-elements in the sense of Stenberg (Definition 2), independently of a concrete mesh family (and hence including $y$-structured macro-elements). In fact one could build an unstructured mesh family converging to a structured one, hence stability is not satisfied when $h \rightarrow 0$. Numerical test 8 shows an example related to this fact. The solution is consider meshes which are uniformly unstructured in the following sense.

Let $\widehat{\mathcal{M}}_{h}$ be a vertex-centered macro-element partitioning a mesh $\mathcal{T}_{h}$. Let $\widehat{M} \in \widehat{\mathcal{M}}_{h}$, let $q$ be its interior vertex and $\left\{T_{1}, \ldots, T_{n}\right\}$ the elements of $\mathcal{T}_{h}$ contained in $\widehat{M}$. For $i=1, \ldots, n-1$, we denote by $\sigma_{i}$ the angle between the positive horizontal semiaxis from $q$ and the common edge of the triangles $T_{i}$ and $T_{i+1}$ of $\widehat{M}$. Similarly, $\sigma_{n}$ is defined using the common edge of $T_{n}$ and $T_{1}$. 


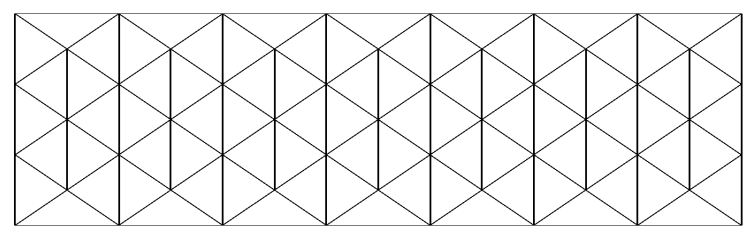

Figure 2: A $x$-structured and $y$-unstructured mesh, where $\left(\mathcal{P}_{1, b}, \mathcal{P}_{1}\right)-\mathcal{P}_{1}$ is Stokes-stable.

Definition 6. A vertex-centered macro-element partitioning $\widehat{\mathcal{M}}_{h}$ is said uniformly $y$-unstructured if there exists $\widehat{\alpha}>0$, independent of $h$, such that $\left|\sin \left(\sigma_{i}\right)\right| \geq \widehat{\alpha}$ for all $i \in\{1, \ldots, n\}$ except, eventually, one $i_{0} \in\{1, \ldots, n\}$. A uniformly $x$-unstructured partitioning is similarly defined (using $\left|\cos \left(\sigma_{i}\right)\right|$ ).

In particular, any uniformly $y$-unstructured macro-element partitioning is composed of $y$ unstructured macro-elements and therefore satisfying regularity condition $(12)$ for $\left(\mathcal{P}_{1, b}, \mathcal{P}_{1}\right)-\mathcal{P}_{1}$, according to local Theorem 3. Now we extend Theorem 3 to a global condition for the stability of $\left(\mathcal{P}_{1, b}, \mathcal{P}_{1}\right)-\mathcal{P}_{1}\left(\right.$ and $\left.\left(\mathcal{P}_{1}, \mathcal{P}_{1, b}\right)-\mathcal{P}_{1}\right)$ in a $2 \mathrm{D}$ mesh $\mathcal{T}_{h}$. Its proof is based on a relaxation of Stenberg's notion of macro-element equivalence while strengthening (respect to Theorem 1 ) the assumptions on $\mathcal{T}_{h}$.

Definition 7. A mesh (family) $\mathcal{T}_{h}$ is said uniformly $y$-unstructured ( $x$-unstructured) if it can be covered by a uniformly $y$-unstructured ( $x$-unstructured) vertex-centered macro-element partitioning $\widehat{\mathcal{M}}_{h}$.

Remark 1. For instance, Figure 2 shows a mesh $\mathcal{T}_{h}$ which is $y$-unstructured, i.e. composed by $y$ unstructured macro-elements $\widehat{M} \in \widehat{\mathcal{M}}_{h}$. In particular one has regularity of $\left(\mathcal{P}_{1, b}, \mathcal{P}_{1}\right)-\mathcal{P}_{1}$ in every $\widehat{M}$. Indeed, $\left|\sin \left(\sigma_{i}\right)\right| \geq \sin (\pi / 4)>0$ for all $\sigma_{i}$ in every $\widehat{M} \in \widehat{\mathcal{M}}_{h}$. If the mesh family $\mathcal{T}_{h}$ preserves this structure when $h$ vanishes, it is uniformly $y$-unstructured.

Theorem 4 (Global stability of $\left(\mathcal{P}_{1, b}, \mathcal{P}_{1}\right)-\mathcal{P}_{1}$ and $\left.\left(\mathcal{P}_{1}, \mathcal{P}_{1, b}\right)-\mathcal{P}_{1}\right)$. Let us consider a triangulation $\mathcal{T}_{h}$ of a domain $\Omega \subset \mathbb{R}^{2}$ satisfying Assumption 1 .

1. If $\mathcal{T}_{h}$ is uniformly $y$-unstructured, then inf-sup condition (6) holds for $\left(\mathcal{P}_{1, b}, \mathcal{P}_{1}\right)-\mathcal{P}_{1}$ elements.

2. If $\mathcal{T}_{h}$ is uniformly $x$-unstructured, then inf-sup condition (6) holds for $\left(\mathcal{P}_{1}, \mathcal{P}_{1, b}\right)-\mathcal{P}_{1}$ elements.

Proof. Let us focus on the first case. It is sufficient to follow the macro-element theory as appears in [Ste90], for the particular case where $\mathcal{T}_{h}$ is a uniformly $y$-unstructured mesh and $\widehat{\mathcal{M}}_{h}$ is a uniform vertex-centered macro-element partitioning, with constant $\widehat{\alpha}$ independent of $h$.

The key is in Lemma 3.2 of [Ste90], whose proof still holds if, instead of equivalent macroelements (in the sense of Stenberg), we consider for each $n=d+1, \ldots, N$ the following " $\widehat{\alpha}$-uniform and $y$-unstructured" equivalence relation (whose equivalence classes will be denoted by $\widehat{\mathcal{E}}_{n, \widehat{\alpha}}$ ): $\widehat{M}^{*}$ and $\widehat{M}$ are equivalent if the following conditions are fulfilled:

a) Both $\widehat{M}^{*}$ and $\widehat{M}$ are in $\widehat{\mathcal{E}}_{n}$ (in particular, they are equivalent in the sense of Stenberg).

b) $\widehat{M}^{*}$ and $\widehat{M}$ are uniformly $y$-unstructured with constant $\widehat{\alpha}$.

Specifically, in Lemma 3.2 of [Ste90], let us fix $\mathcal{E}=\widehat{\mathcal{E}}_{n, \widehat{\alpha}}$. Its proof can be followed line by line, under the following considerations: 


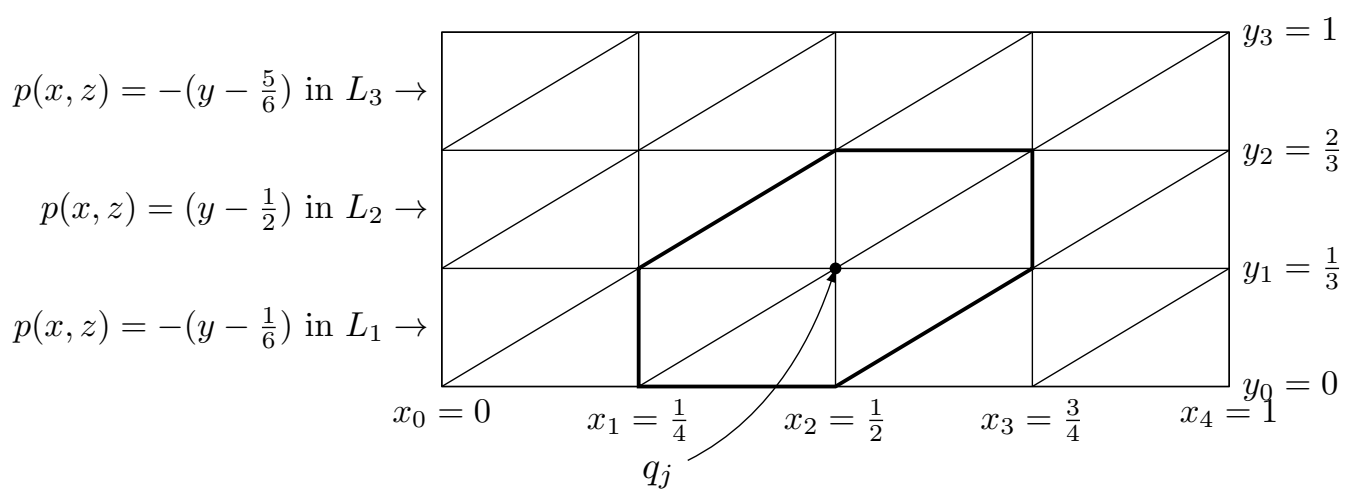

Figure 3: A mesh used as counterexample for showing that $\left(\mathcal{P}_{1, b}, \mathcal{P}_{1}\right)-\mathcal{P}_{1}$ is not stable in structured meshes

1. Every $M \in \widehat{\mathcal{E}}_{n, \widehat{\alpha}}$ is $y$-unstructured, therefore Theorem 3 yields $\beta_{M}>0$ where

$$
\beta_{M}=\min _{\substack{p_{h} \in P_{M} \\\left\|p_{h}\right\|_{M}=1}} \max _{\substack{p_{h} \in \mathbf{W}_{M} \\\left\|\mathbf{w}_{h}\right\|_{1, M}=1}}\left(\nabla \cdot \mathbf{w}_{h}, p_{h}\right)
$$

2. The function $\beta_{M}=\beta_{M}\left(x^{1}, \ldots, x^{k}\right)$, where $x^{i}$ are the vertices of $M$, remains a continuous function.

3. For any macro-element $M$, let $X(M)$ be the set of coordinates of $x^{i}$ (considered as points $\left.X \in \mathbb{R}^{d k}\right)$. Then

$$
\left\{X(M) / M \in \widehat{\mathcal{E}}_{n, \widehat{\alpha}}\right\} \subset\left\{X(M) / M \in \widehat{\mathcal{E}}_{n}\right\} .
$$

Following the proof of Lemma 3.2 of [Ste90], one has that the set on the right hand side is compact. Since the set on left is closed, one can conclude the proof with $\beta_{M} \geq \beta_{n, \widehat{\alpha}}>0$. Since $n<N$ and $\widehat{\alpha}$ if fixed, we have an uniform bound.

Remark 2. Starting from discrete inf-sup condition (6), error estimates analysis of mixed formulations can be applied [BF91], obtaining

$$
\left\|\mathbf{w}-\mathbf{w}_{h}\right\|_{H^{1}}+\left\|p-p_{h}\right\|_{L^{2}} \leq C h \quad \text { and } \quad\left\|\mathbf{w}-\mathbf{w}_{h}\right\|_{L^{2}} \leq C h^{2},
$$

where $C>0$ depends on the $H^{2} \times H^{1}$ norm of $(\mathbf{w}, p)$. It is well-known that second inequality of (19) is based on a duality argument which requires an assumption on the domain (regularity or convexity). Numerical test 3 confirms these statements.

Example 1. It can be demonstrated that in meshes having a strong structure (where every $\widehat{M} \in$ $\widehat{\mathcal{M}}_{h}$ is $x$-structured and y-structured) $L B B$ condition (6) does not hold for $\left(\mathcal{P}_{1, b}, \mathcal{P}_{1}\right)-\mathcal{P}_{1}$ FE. For example, let $\mathcal{T}_{h}$ be the mesh of Figure 3. We are going to show that there exists a family of non null pressures $p_{h} \in P_{h}$ satisfying

$$
\int_{\Omega} \nabla \cdot\left(u_{h}, v_{h}\right) p_{h}=0 \quad \forall u_{h} \in U_{h} \text { and } v_{h} \in V_{h}
$$

hence the discrete Stokes inf-sup condition (6) does not hold for $\left(\mathcal{P}_{1, b}, \mathcal{P}_{1}\right)-\mathcal{P}_{1}$ elements. 
Let $x_{0}, x_{1}, \ldots, x_{k} \in \mathbb{R}$ and $y_{0}, y_{1}, \ldots, y_{l} \in \mathbb{R}$ be two uniform partitionings of two generic intervals $[a, b],[c, d]$ and let $\mathcal{T}_{h}$ be the resulting structured mesh in the rectangle $[a, b] \times[c, d]$, with $l$ vertical layers, $L_{i}=\left(y_{i-1}, y_{i}\right), i=1, \ldots, l$. As suggested by the proof of Theorem 3, let us choose two parameters $\widetilde{a}, \widetilde{c} \in \mathbb{R}, \widetilde{c} \neq 0$ and consider

$$
p_{h}(x, y)=\widetilde{a}+(-1)^{i} \widetilde{c}\left(y-r_{i}\right) \quad \text { if }(x, y) \in L_{i}, \quad \text { for some } r_{i} \in \mathbb{R} .
$$

It is straightforward to test that the condition $p_{h} \in C^{0}(\bar{\Omega})$ can be satisfied selecting $r_{i}=\left(y_{i-1}+y_{i}\right) / 2$ and, in this case, if $\widetilde{a}=0$ then $p_{h} \in P_{h}$ because $\int_{\Omega} p_{h}=0$. Figure 3 shows a particular case, with $\widetilde{c}=1$.

Let $q_{j}$ and $\phi_{j}, j=1, \ldots, N=(k-1)(l-1)$ be respectively the vertices in the interior of $\mathcal{T}_{h}$ and the associated $\mathcal{P}_{1}$ basis functions. As $\partial_{x} p_{h}=0$, if $\left(u_{h}, v_{h}\right) \in U_{h} \times V_{h}$ then

$$
\int_{\Omega} \nabla \cdot\left(u_{h}, v_{h}\right) p_{h}=-\int_{\Omega} v_{h} \partial_{y} p_{h}=\sum_{j=1}^{N} a_{j} \int_{\Omega} \phi_{j} \partial_{y} p_{h}
$$

for some $a_{j} \in \mathbb{R}$. For all $j=1, \ldots, N$, let $T_{i j}(i=1, \ldots, 6)$ be the triangles adjacent to the interior vertex $q_{j}$, forming a macro-element like in Figure 3. Without loss of generality, we can suppose that $T_{1 j}, T_{2 j}$ and $T_{3 j}$ are laying in an even layer, $T_{4 j}, T_{5 j}$ and $T_{6 j}$ in an odd one and $\left|T_{i j}\right|=1$ for all $i$. Then,

$$
\int_{\Omega} \phi_{j} \partial_{y} p_{h}=\sum_{i=1}^{3} \widetilde{c} \int_{T_{i j}} \phi_{j}-\sum_{i=4}^{6} \widetilde{c} \int_{T_{i j}} \phi_{j}=\frac{\widetilde{c}}{3}\left(\sum_{i=1}^{3}\left|T_{i j}\right|-\sum_{i=4}^{6}\left|T_{i j}\right|\right)=0 .
$$

Therefore $\int_{\Omega} \phi_{j} \partial_{y} p_{h}=0$ for all $j=1, \ldots, N$ and (20) holds.

This example is also valid to prove that $\left(\mathcal{P}_{1}, \mathcal{P}_{1, b}\right)-\mathcal{P}_{1}$ is not stable in structured meshes.

Remark 3. Theorem 4 provides a sufficient but not necessary condition for the (global) stability of $\left(\mathcal{P}_{1}, \mathcal{P}_{1, b}\right)-\mathcal{P}_{1}\left(\right.$ and $\left.\left(\mathcal{P}_{1, b}, \mathcal{P}_{1}\right)-\mathcal{P}_{1}\right)$. Indeed some other (not vertex-centered) macro-element families may also conduce to stability.

In order to ensure that $\mathcal{T}_{h}$ is uniformly $x$-unstructured (and therefore $\left(\mathcal{P}_{1}, \mathcal{P}_{1, b}\right)-\mathcal{P}_{1}$ is stable), the following mesh post-processing algorithm may be applied (similarly for $\left(\mathcal{P}_{1, b}, \mathcal{P}_{1}\right)-\mathcal{P}_{1}$ in $y-$ unstructured meshes):

\section{Algorithm 1.}

- Fix a horizontal "unstructuring factor" $r \in(0,1)$ and define $h_{r}=r \cdot h$, where $h$ is the mesh size. For instance, $r=0.15$ in our experiments. If $r$ is too small, mesh might not be unstructured enough and if $r$ is too big, mesh is too deformed.

- For each interior vertex, $q_{0}$, let $\widehat{M}_{q_{0}}$ be the macro element centered in $q_{0}$ and let $q_{1}, \ldots, q_{\mathrm{n}_{v}}$ be the other vertices in $\widehat{M}_{q_{0}}$. We denote $q_{i}=\left(q_{i}^{x}, q_{i}^{y}\right)$ its coordinates.

- For each $i=1, \ldots, \mathrm{n}_{v}$, let $d_{i}=q_{i}^{x}-q_{0}^{x}$. If $\left|d_{i}\right|<h_{r}$ (i.e. the edge $q_{0} q_{i}$ is "almost vertical") then:

* For each $j=i+1, \ldots, \mathrm{n}_{v}$, if $\left|q_{j}^{x}-q_{0}^{x}\right|<h_{r}$ (then $\widehat{M}_{q_{0}}$ is "almost $x$-structured"):

- If $d_{i}>0$ :

$$
q_{0}^{x}=q_{0}^{x}-\left(h_{r}-d_{i}\right), \quad\left(\text { move } q_{0} \text { to the left }\right)
$$

- else:

$$
q_{0}^{x}=q_{0}^{x}+\left(h_{r}+d_{i}\right), \quad \text { (move } q_{0} \text { to the right) }
$$


See numerical test 4 for a practical experiment. Note that this algorithm ensures that the resulting mesh is uniformly $x$-unstructured. Indeed, if for some $\widehat{M}_{q_{0}}$ one has two edges, $q_{0} q_{i}$ and $q_{0} q_{j}$, "almost vertical", $q_{0}$ is displaced and then $\left|q_{0}^{x}-q_{i}^{x}\right|=h_{r}$. In consequence, $\widehat{M}_{q_{0}}$ and also $\widehat{M}_{q_{i}}$ (if $q_{i}$ is an interior vertex) are $x$-unstructured. Note that, in particular, $q_{i}$ shall not be displaced in following iterations and thus $\widehat{M}_{q_{0}}$ remains $x$-unstructured.

\subsection{The $3 D$ case}

Results obtained in Section 3.1 are now generalized to $3 D$ domains. Specifically, we aboard the stability of the FE spaces which arise when one (or two) of the three components of a $\mathcal{P}_{1}$ continuous approximation of velocity field is enriched with a bubble per element. Thus Theorem 3 is extended to the $3 D$ in two ways, depending on whether two or one of the components are enriched.

First possibility: $\left(\mathcal{P}_{1}, \mathcal{P}_{1, b}, \mathcal{P}_{1, b}\right)-\mathcal{P}_{1}$

First we focus on the approximation of the discrete Stokes equations (4)-(5) in the velocity space $\mathbf{W}_{h}=U_{h} \times \mathbf{V}_{h}$, being $U_{h}$ a $\mathcal{P}_{1}$ (continuous) space and $\mathbf{V}_{h}$ the product of two $\mathcal{P}_{1, b}$ (continuous) spaces, $V_{1} \times V_{2}$. Also $P_{h}$ is taken as a $\mathcal{P}_{1}$ continuous space, as defined in (11).

Let $\mathcal{T}_{h}$ be a $3 D$ mesh composed of tetrahedrons, denoted as $K_{i}$, satisfying Assumption 1 and let $\widehat{\mathcal{M}}_{h}$ be the associated $3 D$ vertex-centered macro-element partitioning. Let $\mathbf{W}_{0, M}$ be the space of functions $\mathbf{w}_{h}=\left(u_{h}, v_{h}^{1}, v_{h}^{2}\right) \in H_{0}^{1}(M)^{3} \cap C^{0}(\bar{M})^{3}$ such that $u_{h}$ is a $\mathcal{P}_{1}$ function in each $K \subset M$ while $v_{h}^{1}, v_{h}^{2}$ are $\mathcal{P}_{1, b}$ functions and let $P_{M}$ be the space of $\mathcal{P}_{1}$ continuous functions in each tetrahedron of $M$. Due to Theorem 1 and Lemma 2, a sufficient condition for the stability of $\left(\mathcal{P}_{1}, \mathcal{P}_{1, b}, \mathcal{P}_{1, b}\right)-\mathcal{P}_{1}$ in $\mathcal{T}_{h}$ is the following $3 D$ condition of regularity in macro-elements, for all $\widehat{M} \in \widehat{\mathcal{M}}_{h}$ :

$$
\begin{aligned}
& \text { The set } N_{\widehat{M}}=\left\{p_{h} \in P_{\widehat{M}} / \int_{\widehat{M}} p_{h} \nabla \cdot \mathbf{w}_{h}=0, \forall \mathbf{w}_{h} \in \mathbf{W}_{0, \widehat{M}}\right\} \\
& \text { only consists of the functions which are constant on } \widehat{M} \text {. }
\end{aligned}
$$

Next we are going to prove the following local regularity result, which constitutes a $3 D$ version of Theorem 3.

Theorem 5. Let $\widehat{M}$ be a macro-element in a vertex-centered partitioning $\widehat{\mathcal{M}}_{h}$. Then the combination $\left(\mathcal{P}_{1}, \mathcal{P}_{1, b}, \mathcal{P}_{1, b}\right)-\mathcal{P}_{1}$ is regular in $\widehat{M}$ if and only if $\widehat{M}$ is $x$-unstructured. The cases $\left(\mathcal{P}_{1, b}, \mathcal{P}_{1}, \mathcal{P}_{1, b}\right)-\mathcal{P}_{1}$ and $\left(\mathcal{P}_{1, b}, \mathcal{P}_{1, b}, \mathcal{P}_{1}\right)-\mathcal{P}_{1}$ are symmetric.

Proof.

$\Leftarrow$ Let us define $p_{h} \in N_{\widehat{M}}$ with $\left.p_{h}\right|_{K_{i}}=a_{i}+b_{i} x+c_{i} y+d_{i} z$, for each $K_{i} \subset \widehat{M}$. Let $q_{r}, r=1, \ldots \mathrm{n}_{v}$, be the vertices on $\partial \widehat{M}$, let $q_{0}$ the only vertex in the interior of $\widehat{M}$ and let $r_{i}, i=1, \ldots, n_{K}$ be the bubble degrees of freedom (where $n_{K}$ is the number of tetrahedrons in $\widehat{M}$ ).

If we choose $u_{h}=0, v_{h}^{2}=0$ and $v_{h}^{1}$ is equal to 1 in the barycentre of a tetrahedron $K_{i}$ while is 0 in all other degrees of freedom, we have

$$
0=\int_{\widehat{M}} \partial_{y} v_{h}^{1} p_{h}=-\int_{\widehat{M}} v_{h}^{1} \partial_{y} p_{h}=-c_{i} \int_{K_{i}} v_{h}^{1}
$$

Then $c_{i}=0$ for all $i=1, \ldots, n_{K}$. Interchanging the roles of $v_{h}^{1}$ and $v_{h}^{2}$, we also deduce $d_{i}=0$ for all $i=1, \ldots, n_{K}$. Therefore, $\left.p_{h}\right|_{K_{i}}=a_{i}+b_{i} x$. 
Now, supposing that $q_{0}=(0,0,0)$ and applying the continuity of $p_{h}$ in $q_{0}$, we conclude that exists $a \in \mathbb{R}$ such that $a_{1}=\ldots=a_{n_{K}}=a$. Then, if $T_{i j}$ denotes the (triangular) face common to two tetrahedrons, $K_{i}$ and $K_{j}$, the continuity of $p_{h}$ in $T_{i j}$ implies

$$
\left\{\begin{array}{l}
x_{1}\left(b_{i}-b_{j}\right)=0, \\
x_{2}\left(b_{i}-b_{j}\right)=0,
\end{array}\right.
$$

where $x_{1}$ and $x_{2}$ are the respective $x$ coordinates of the two vertices which, besides $q_{0}$, define $T_{i j}$.

For every $i$ and $j$ such that the face $T_{i j}$ is not included in the plane $x=0$, then $x_{1}$ or $x_{2}$ is not zero, hence $b_{i}=b_{j}$. On the other hand, by hypothesis, there is no plane $x=C$ whose intersection with $\widehat{M}$ is formed by faces of tetrahedrons. Then any two tetrahedrons $K_{i}$ and $K_{j}$ in $\widehat{M}$ can be connected by a chain of faces satisfying the property of not inclusion in the plane $x=0$, hence $b_{i}=b_{j}=b$ for all $i, j=1, \ldots, n_{K}$, for some $b \in \mathbb{R}$, and $p_{h}=a+b x$.

It remains to prove $b=0$. Let us choose $v_{h}^{1}=v_{h}^{2}=0$ and $u_{h}$ equal to 1 in $q_{0}$ and 0 in all other vertices. Then

$$
0=\int_{\widehat{M}} \partial_{x} u_{h} p_{h}=-\int_{\widehat{M}} u_{h} \partial_{x} p_{h}=-b \int_{\widehat{M}} u_{h},
$$

hence $b=0$, what means $p$ is constant on $\widehat{M}$ and the macro-element condition (22) holds.

For the reciprocal implication, we use a reduction to absurdity argument. Let $\widehat{M} \in \widehat{\mathcal{M}}_{h}$ with $q_{0}=(0,0,0)$ its only interior vertex and let us suppose that $\widehat{M}$ can be split by the plane $x=0$.

Let $\widehat{M}^{+}=\{(x, y, z) \in \widehat{M} / x>0\}$ and $\widehat{M}^{-}=\{(x, y, z) \in \widehat{M} / x<0\}$ and, for any parameters $a$ and $b \in \mathbb{R}, b \neq 0$, let us consider the family of (not constant) pressures of $P_{\widehat{M}}$ :

$$
p_{h}(x, y, z)= \begin{cases}a-\frac{b}{\left|\widehat{M}^{-}\right|} x & \text { if } x \leq 0, \\ a+\frac{b}{\left|\widehat{M}^{+}\right|} x & \text { if } x>0 .\end{cases}
$$

Reasoning like in the proof of Theorem 3 , it is not difficult to show that any $p_{h}$ in this family satisfies $\int_{\widehat{M}} p_{h} \nabla \cdot \mathbf{w}_{h}=0$ for all $\mathbf{w}_{h}$ in $\mathbf{W}_{0, \widehat{M}}$, hence $\left(\mathcal{P}_{1}, \mathcal{P}_{1, b}, \mathcal{P}_{1, b}\right)-\mathcal{P}_{1}$ is not regular in $\widehat{M}$.

Remark 4. Theorem 5 can be applied (owing to Theorem 1 and Lemma 2) in order to provide a sufficient condition for the stability of $\left(\mathcal{P}_{1}, \mathcal{P}_{1, b}, \mathcal{P}_{1, b}\right)-\mathcal{P}_{1}$ in uniformly unstructured $3 D$ meshes. Indeed, a global result similar to Theorem 4 can be enounced, including also the cases $\left(\mathcal{P}_{1, b}, \mathcal{P}_{1}, \mathcal{P}_{1, b}\right)-\mathcal{P}_{1}$ and $\left(\mathcal{P}_{1, b}, \mathcal{P}_{1, b}, \mathcal{P}_{1}\right)-\mathcal{P}_{1}$

Finally, as commented in Remark 2, our numerical experiments suggest stability in usual unstructured meshes. In any case, Algorithm 1 can be generalized and applied for ensure stability. Error estimates (19) can be extended to this $3 D$ case.

Second possibility: $\left(\mathcal{P}_{1}, \mathcal{P}_{1}, \mathcal{P}_{1, b}\right)-\mathcal{P}_{1}$

Next, two components of the velocity field are being left without enrichment, while the remaining component is enriched with bubbles. To simplify our exposition, we focus on the case where bubbles are added "in the vertical direction", but not "in horizontal directions". Namely additional bubbles are inserted in the $z$ component of velocity, but not in $x$ or $y$ components, obtaining $\left(\mathcal{P}_{1}, \mathcal{P}_{1}, \mathcal{P}_{1, b}\right)-\mathcal{P}_{1}$ FE (the other cases $\left(\mathcal{P}_{1, b}, \mathcal{P}_{1}, \mathcal{P}_{1}\right)-\mathcal{P}_{1}$ and $\left(\mathcal{P}_{1}, \mathcal{P}_{1, b}, \mathcal{P}_{1}\right)-\mathcal{P}_{1}$ are symmetric).

Generalizing the $2 \mathrm{D}$ case, the idea that we are going to formalize is: $\left(\mathcal{P}_{1}, \mathcal{P}_{1}, \mathcal{P}_{1, b}\right)-\mathcal{P}_{1}$ is stable in unstructured in directions $(x, y)$ macro-elements. Namely, macro-elements which cannot be split 
by planes (more specifically semiplanes, see Theorem 8) with equations $a x+b y+c=0$ (orthogonal to the horizontal plane $z=0$ ). That is, the key is: vertical semiplanes.

Let $\mathcal{T}_{h}$ be a $3 D$ mesh which is composed of tetrahedrons and satisfies Assumption 1. Let us consider $\mathbf{W}_{h}=\mathbf{U}_{h} \times V_{h}$, were $\mathbf{U}_{h}$ is the product of two $\mathcal{P}_{1}$ (continuous) spaces, $U_{1}$ and $U_{2}$, while $V_{h}$ is a $\mathcal{P}_{1, b}$ (continuous) space. Again, the macro-element regularity condition (22) is sufficient for the regularity of $\left(\mathcal{P}_{1}, \mathcal{P}_{1}, \mathcal{P}_{1, b}\right)-\mathcal{P}_{1}$ in any macro-element $\widehat{M} \in \widehat{\mathcal{M}}_{h}$ where, in this case, $\mathbf{W}_{0, \widehat{M}}$ is the space of functions $\mathbf{w}_{h}=\left(u_{h}^{1}, u_{h}^{2}, v_{h}\right) \in H_{0}^{1}(\widehat{M})^{3} \cap C^{0}(\widehat{M})^{3}$ such that $u_{h}^{1}$ and $u_{h}^{2}$ are $\mathcal{P}_{1}$ while $v_{h}$ is $\mathcal{P}_{1, b}$. The following geometrical results are going to be useful:

Lemma 6. Let $\Pi$ a plane containing a triangle, $T$, whose vertices are $q_{0}=\left(x_{0}, y_{0}, z_{0}\right), q_{1}=$ $\left(x_{1}, y_{1}, z_{1}\right)$ and $q_{2}=\left(x_{2}, y_{2}, z_{2}\right)$. Then $\Pi$ is orthogonal to the plane $z=0$ if and only if

$$
\left|\begin{array}{ll}
x_{1}-x_{0} & y_{1}-y_{0} \\
x_{2}-x_{0} & y_{2}-y_{0}
\end{array}\right|=0
$$

Proof. If $\Pi$ is a plane containing the triangle $T$, then it is defined by $q_{0}$ and the vectors $v_{1}=$ $\left(x_{1}-x_{0}, y_{1}-y_{0}, z_{1}-z_{0}\right)$ and $v_{1}=\left(x_{2}-x_{0}, y_{2}-y_{0}, z_{2}-z_{0}\right)$. Then, $\Pi$ is orthogonal to the plane $z=0$ if and only if the normal vector, $(0,0,1)$, to the plane $z=0$ is a linear combination of $v_{1}$ and $v_{2}$, that is

$$
\left|\begin{array}{ccc}
0 & 0 & 1 \\
x_{1}-x_{0} & y_{1}-y_{0} & z_{1}-z_{0} \\
x_{1}-x_{0} & y_{2}-y_{0} & z_{2}-z_{0}
\end{array}\right|=0
$$

and this equation is equivalent to (23).

Lemma 7. Let $\widehat{M} \in \widehat{\mathcal{M}}_{h}$. Let $M^{\prime}=K_{1} \cup \ldots \cup K_{n}$, where $K_{1}, \ldots, K_{n}$ are tetrahedrons contained in $\widehat{M}$ which are connected by a chain of (triangular) faces which are not orthogonal to the plane $z=0$ (namely, not vertical faces). If $p_{h} \in N_{\widehat{M}}$, there exists $a, b^{\prime}, c^{\prime} \in \mathbb{R}$ (with a independent on $M^{\prime}$ ) such that $\left.p_{h}\right|_{M^{\prime}}=a+b^{\prime} x+c^{\prime} y$.

Proof. Let $p_{h} \in N_{\widehat{M}}$, with $\left.p\right|_{K_{i}}=a_{i}+b_{i} x+c_{i} y+d_{i} z$ in each tetrahedron $K_{i}$. We can consider $v_{h}=1$ in the barycentre of $K_{i}$ and $v_{h}=0$ in all other degrees of freedom, while $u_{h}^{1}=u_{h}^{2}=0$ in $\widehat{M}$. Similarly to the proof of Theorem 5 , we get $d_{i}=0$ for all $i=1, \ldots, n_{K}$. Supposing, without loss of generality, that the common vertex in $\widehat{M}$ is $q_{0}=(0,0,0)$ and using the continuity of $p_{h}$ in $q_{0}$ we have $a_{1}=\ldots=a_{n_{K}}=a$, for some $a \in \mathbb{R}$, then

$$
\left.p\right|_{K_{i}}=a+b_{i} x+c_{i} y, \quad \forall i=1, \ldots, n_{K} .
$$

If $T_{i j}$ is the common face of two tetrahedrons $K_{i}$ and $K_{j}$, and $q_{1}=\left(x_{1}, y_{1}, z_{1}\right), q_{2}=\left(x_{2}, y_{2}, z_{2}\right)$ are, besides $q_{0}$, its vertices, the continuity of $p_{h}$ in $T_{i j}$ is equivalent to

$$
\left\{\begin{array}{l}
x_{1}\left(b_{i}-b_{j}\right)+y_{1}\left(c_{i}-c_{j}\right)=0, \\
x_{2}\left(b_{i}-b_{j}\right)+y_{2}\left(c_{i}-c_{j}\right)=0 .
\end{array}\right.
$$

If $T_{i j}$ is not vertical, from Lemma 6 (with $x_{0}=y_{0}=0$ )

$$
\left|\begin{array}{ll}
x_{1} & y_{1} \\
x_{2} & y_{2}
\end{array}\right| \neq 0
$$

and then

$$
b_{i}-b_{j}=0 \quad \text { and } \quad c_{i}-c_{j}=0 .
$$


Finally, if the tetrahedrons $K_{1}, \ldots, K_{n}$ can be connected through a chain of faces, $T_{1,2}, T_{2,3}, \ldots$, $T_{n-1, n}$, that are not orthogonal to the plane $z=0$, then $b_{1}=b_{2}=\ldots=b_{n}=b^{\prime}$ and $c_{1}=c_{2}=\ldots=$ $c_{n}=c^{\prime}$, for some $b^{\prime}, c^{\prime} \in \mathbb{R}$.

For the following result, we use the concept of splitting a macro-element by a set of semi-planes. This splitting concept can be defined in the sense of Definition 4.

Theorem 8. Let $\widehat{M} \in \widehat{\mathcal{M}}_{h}$ with one only interior vertex, $q_{0}$. Let $r_{0}$ be the vertical straight line through $q_{0}$ and let $\mathcal{F}$ be the family of the (vertical) semi-planes bounded by $r_{0}$.

1. If $\widehat{M}$ cannot be split by semi-planes of $\mathcal{F}$, then $\left(\mathcal{P}_{1}, \mathcal{P}_{1}, \mathcal{P}_{1 b}\right)-\mathcal{P}_{1}$ is regular in $\widehat{M}$.

2. If $\widehat{M}$ can be split by only two semi-planes of $\mathcal{F}$, then $\left(\mathcal{P}_{1}, \mathcal{P}_{1}, \mathcal{P}_{1 b}\right)-\mathcal{P}_{1}$ is regular in $\widehat{M}$ if and only if these two semi-planes are not aligned (i.e. they do not form a vertical plane splitting $\widehat{M})$.

3. If $\widehat{M}$ can be split by more than two semi-planes of $\mathcal{F}$, then $\left(\mathcal{P}_{1}, \mathcal{P}_{1}, \mathcal{P}_{1 b}\right)-\mathcal{P}_{1}$ is not regular in $\widehat{M}$.

Proof. Let $p_{h}$ in $N_{\widehat{M}}$ with $\left.p_{h}\right|_{T_{i}}=a_{i}+b_{i} x+c_{i} y+d_{i} z, i=1, \ldots n_{K}$. Without loss of generality, we can assume $q_{0}=(0,0,0)$. Then, by continuity in $q_{0}, a_{1}=\ldots=a_{n}$. Let us choose $u_{h}^{2}=v_{h}=0$ in $\widehat{M}$ while $u_{h}^{1}$ is equal to 1 in $q_{0}$ and 0 in all other degrees of freedom. Using the quadrature formula defined in the vertices of tetrahedrons (exact in $\mathcal{P}_{1}$ ), the following condition is satisfied:

$$
0=\int_{\widehat{M}} \partial_{x} u_{h}^{1} p_{h}=-\sum_{i=1}^{n_{K}}\left|K_{i}\right| b_{i} .
$$

Applying the same argument to $u_{h}^{1}=v_{h}=0$ while $u_{h}^{2}$ is equal to 1 in $q_{0}$ and 0 in all other degrees of freedom:

$$
0=\int_{\widehat{M}} \partial_{y} u_{h}^{2} p_{h}=-\sum_{i=1}^{n_{K}}\left|K_{i}\right| c_{i} .
$$

1. Since $\widehat{M}$ cannot be split by semi-planes of $\mathcal{F}$, then any two tetrahedrons of $\widehat{M}$ can be connected by a chain of not vertical faces. Applying Lemma 7 to $M^{\prime}=\widehat{M}$, one has that $p_{h}$ can be written as $p_{h}=a+b x+c y$. Then, taking into account (24) and (25), b=0 and $c=0$, hence $p$ is constant on $\widehat{M}$ and the macro-element condition (22) holds.

2. If $\widehat{M}$ can be split only by two semi-planes, $\Pi_{1}$ and $\Pi_{2}$ of $\mathcal{F}$ into two regions $M_{1}^{\prime}$ and $M_{2}^{\prime}$ then (according to Lemma 7 ), there exist $b_{1}, c_{1}, b_{2}, c_{2} \in \mathbb{R}$ such that

$$
\begin{aligned}
\left.p_{h}\right|_{M_{1}^{\prime} u} & =a+b_{1} x+c_{1} y, \\
\left.p_{h}\right|_{M_{2}^{\prime}} & =a+b_{2} x+c_{2} y .
\end{aligned}
$$

Let $q_{0}=(0,0,0), q_{1}=\left(x_{1}, y_{1}, z_{1}\right)$ and $q_{2}=\left(x_{2}, y_{2}, z_{1}\right)$ be the vertices of an interior vertical face $T \subset \Pi_{1}$. The continuity of $p_{h}$ in $T$ means:

$$
\begin{aligned}
& x_{1}\left(b_{1}-b_{2}\right)+y_{1}\left(c_{1}-c_{2}\right)=0, \\
& x_{2}\left(b_{1}-b_{2}\right)+y_{2}\left(c_{1}-c_{2}\right)=0 .
\end{aligned}
$$


But, according to Lemma 6,

$$
\left|\begin{array}{ll}
x_{1} & y_{1} \\
x_{2} & y_{2}
\end{array}\right|=0
$$

then (26) and (27) are reduced to one only equation, e.g. (27).

Similarly, if $q_{0}=(0,0,0), \widetilde{q}_{1}=\left(\widetilde{x}_{1}, \widetilde{y}_{1}, \widetilde{z}_{1}\right)$ and $\widetilde{q}_{2}=\left(\widetilde{x}_{2}, \widetilde{y}_{2}, \widetilde{z}_{1}\right)$ are the vertices of an interior vertical face $\widetilde{T} \subset \Pi_{2}$, one only equation is obtained from the continuity of $p_{h}$ in $\widetilde{T}$ :

$$
\widetilde{x}_{1}\left(b_{1}-b_{2}\right)+\widetilde{y}_{2}\left(c_{1}-c_{2}\right)=0 .
$$

$\Leftarrow$ If the vertical semi-planes $\Pi_{1}$ and $\Pi_{2}$ are not aligned then the projections of $\left(x_{1}, y_{1}, z_{1}\right) \in \Pi_{1}$ and $\left(\widetilde{x}_{1}, \widetilde{y}_{1}, \widetilde{z}_{1}\right) \in \Pi_{2}$ in $z=0$ are not aligned, that is:

$$
\left|\begin{array}{ll}
x_{1} & y_{1} \\
\widetilde{x}_{1} & \widetilde{y}_{2}
\end{array}\right| \neq 0
$$

Hence, from (27)-(28), $b_{1}=b_{2}$ and $c_{1}=c_{2}$. Using (24)-(25), we conclude $b_{1}=b_{2}=c_{1}=c_{2}=0$, and $p_{h}=a$ in $\widehat{M}$.

$\Rightarrow$ If $\Pi_{1}$ and $\Pi_{2}$ are aligned, (27) and (28) are reduced to one only equation which is not enough to conclude $b_{1}=b_{2}=c_{1}=c_{2}=0$. In fact, counterexamples of not constant pressures of $N_{\widehat{M}}$ satisfying $b_{1} \neq b_{2}$ or $c_{1} \neq c_{2}$, can be obtained similarly to the counterexample given in the proof of Theorem 5 .

3. Let $M_{1}^{\prime}, \ldots, M_{n}^{\prime}$ be regions resulting from splitting $\widehat{M}$ by $n$ semi-planes $(n>2), \Pi_{1}, \ldots, \Pi_{n}$ of $\mathcal{F}$. Arguing as in the previous case one has that, for each $i=1, \ldots, n,\left.p_{h}\right|_{M_{i}^{\prime}}=a+b_{i} x+c_{i} y$ and the continuity of $p_{h}$ in $\Pi_{i}$ can be reduced to one only equation:

$$
x_{i}\left(b_{i}-b_{i+1}\right)+y_{i}\left(c_{i}-c_{i+1}\right)=0,
$$

where $q_{i}=\left(x_{i}, y_{i}, z_{i}\right) \in \Pi_{i}$. Therefore, we have at most $n$ independent equations with $2 n$ unknowns, $b_{i}$ and $c_{i}$. Since $n>2$, the equations (24) and (25) are not enough to conclude $b_{i}=c_{i}=0$ for all $i=1, \ldots, n$, because we have at most $n+2$ independent equations but $2 n$ unknowns (and $n+2<2 n$ if $n>2$ ).

Remark 5. Using Theorem 1 and Lemma 2, the macro-element regularity result given in Theorem 8 can be used as a sufficient condition assuring the stability of $\left(\mathcal{P}_{1}, \mathcal{P}_{1}, \mathcal{P}_{1 b}\right)-\mathcal{P}_{1}$ in $3 D$ meshes that are uniformly $z$-unstructured, in the sense of meshes which uniformly do not present a local structure like the one discussed in Theorem 8 (splitting by vertical semi-planes). In practice, one can hope that the unstructured $3 D$ meshes obtained with usual software tools present this property but, in any case, mesh may be tested and eventually slightly modified (via a generalization of Algorithm 1) for ensuring stability.

\subsection{Numerical simulations}

Some computational simulations are provided, which agree with the numerical analysis of previous sections.

Test $1\left(\left(\mathcal{P}_{1, b}, \mathcal{P}_{1}\right)-\mathcal{P}_{1}\right.$ and the cavity test in a $x$-structured and $y$-unstructured mesh). As first numerical test we have chosen, in the unit square $\Omega=(0,1)^{2}$, a $x$-structured and $y$ unstructured mesh like the one shown in Figure 2, where the stability of $\left(\mathcal{P}_{1, b}, \mathcal{P}_{1}\right)-\mathcal{P}_{1}$ is guaranteed 


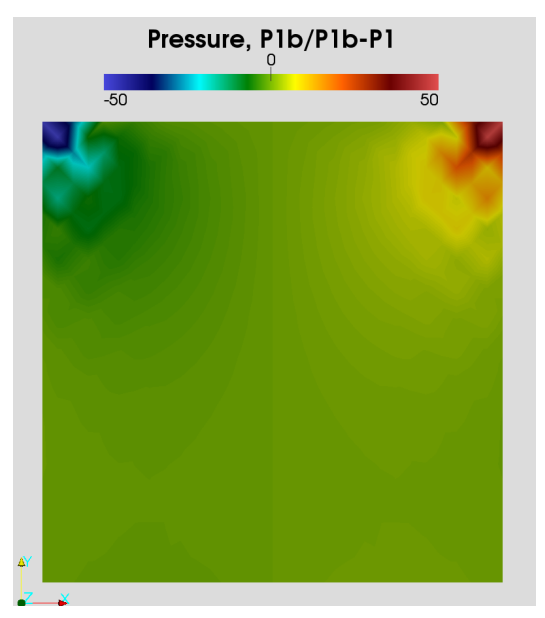

(a) $\left(\mathcal{P}_{1, b}, \mathcal{P}_{1, b}\right)-\mathcal{P}_{1}$

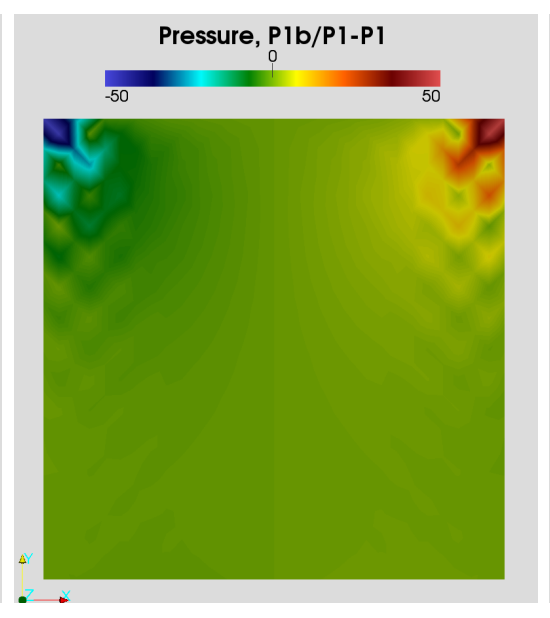

(b) $\left(\mathcal{P}_{1, b}, \mathcal{P}_{1}\right)-\mathcal{P}_{1}$.

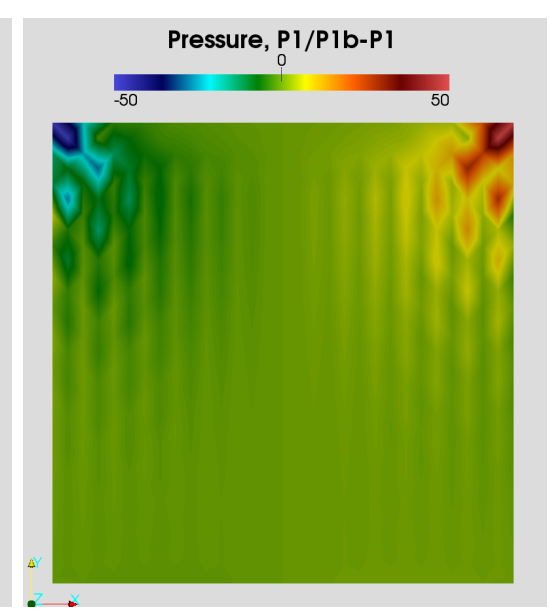

(c) $\left(\mathcal{P}_{1}, \mathcal{P}_{1, b}\right)-\mathcal{P}_{1}$.

Figure 4: Pressure contour plots in a $y$-unstructured mesh (lid-driven cavity test).

(see Remark 1). We have approximated the solution of the Stokes problem (1)-(3) with Dirichlet conditions $u=1$ on the top boundary, $u=0$ on the rest of $\partial \Omega$ and $v=0$ on all $\partial \Omega$.

For the practical implementation, that specific mesh is generated (with $15 \times 15$ edges, i.e. $h \simeq$ 0.666) by a script programmed using the Python language, which generates a mesh file using the format of the mesh generator Gmsh [GR09]. This file is imported by the Stokes solver, which has been programmed using the FEniCS FE suite $\left[\mathrm{LMW}^{+} 12\right]$, taking advantage of the facilities offered by this tool for setting the boundary conditions on this specific mesh.

The program has been run for three choices: $\left(\mathcal{P}_{1, b}, \mathcal{P}_{1, b}\right)-\mathcal{P}_{1},\left(\mathcal{P}_{1, b}, \mathcal{P}_{1}\right)-\mathcal{P}_{1}$ and $\left(\mathcal{P}_{1}, \mathcal{P}_{1, b}\right)-\mathcal{P}_{1}$. As expected, the last case presents the worst behaviour for the pressure, suggesting the instability of $\left(\mathcal{P}_{1}, \mathcal{P}_{1, b}\right)-\mathcal{P}_{1}$ in these $x$-structured meshes (Figure $4 \mathrm{c}$ ). On the other hand, the results for $\left(\mathcal{P}_{1}, \mathcal{P}_{1, b}\right)-\mathcal{P}_{1}$ are similar to the mini-element $\left(\mathcal{P}_{1, b}, \mathcal{P}_{1, b}\right)-\mathcal{P}_{1}$ (Figures $4 \mathrm{~b}$ and $4 \mathrm{a}$, respectively), as expected from the stability of $\left(\mathcal{P}_{1, b}, \mathcal{P}_{1}\right)-\mathcal{P}_{1}$ in $y$-unstructured meshes.

Notice that in this test (and in the next ones below) the Stokes mixed scheme (4)-(5) has been slightly altered by introducing a standard pressure penalization in the divergence equation:

$$
\int_{\Omega}\left(\partial_{x} u+\partial_{y} v\right) \bar{p}+\varepsilon \int_{\Omega} p \bar{p}=0, \quad \forall p \in P_{h},
$$

where $\varepsilon$ is a small penalization parameter (in this example, we have selected $\varepsilon=10^{-10}$ ). With this approximation, the condition $\int_{\Omega} p=0$ is satisfied implicitly (we obtain $\int_{\Omega} p=-5.12 \cdot 10^{-8}$ ) and hence it is no longer necessary to include it in the definition of $P_{h}$. Then, we can take $P_{h}=\left\{p_{h} \in\right.$ $\left.C^{0}(\bar{\Omega}) /\left.p\right|_{T} \in \mathcal{P}_{1}, \quad \forall T \in \mathcal{T}_{h}\right\}$.

Test $2\left(\left(\mathcal{P}_{1, b}, \mathcal{P}_{1}\right)-\mathcal{P}_{1}\right.$ and the cavity test $)$. As the second numerical test, the $2 \mathrm{D}$ Stokes problem (1)-(3) has been solved in the squared domain $\Omega=(0,1)^{2} \subset \mathbb{R}^{2}$, using $\left(\mathcal{P}_{1, b}, \mathcal{P}_{1}\right)-\mathcal{P}_{1}$ FE in two different meshes: an unstructured mesh with 3,792 triangles (built with 20 edges on each boundary segment, see Figure 5a) and a structured mesh with the same number of boundary edges (Figure 6a). Then, in both cases $h \simeq 1 / 20$.

We have fixed the Neumann condition $\nu \frac{\partial u}{\partial z}=1$ on the top boundary, while $u=0$ on the rest of $\partial \Omega$ and $v=0$ on all $\partial \Omega$. In this case, the implementation has been developed in FreeFem ++ [PHLHM].

In both simulations (unstructured and structured mesh) the velocity filed present similar behavior (Figures 5b and 6b). But the pressure unknown, that is correct in the unstructured case 


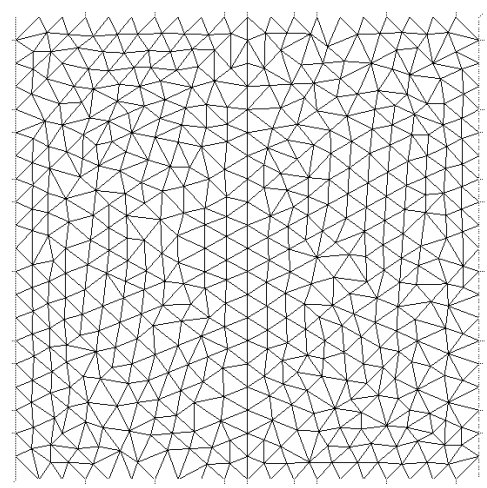

(a) Unstructured mesh.

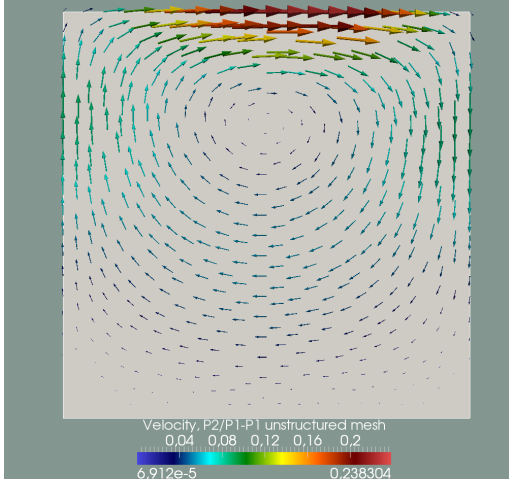

(b) Velocity field.

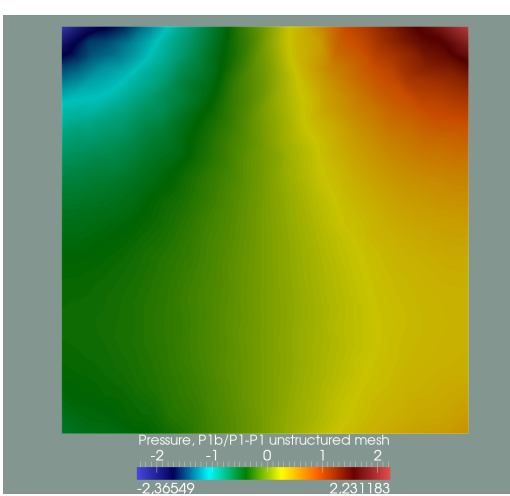

(c) Pressure.

Figure 5: $\left(\mathcal{P}_{1, b}, \mathcal{P}_{1}\right)-\mathcal{P}_{1}$ elements in a unstructured mesh.

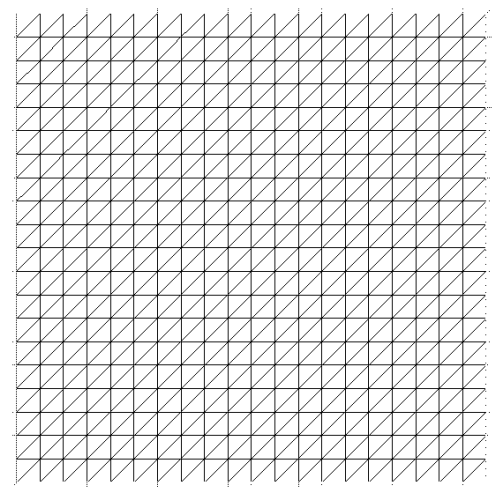

(a) Structured mesh.

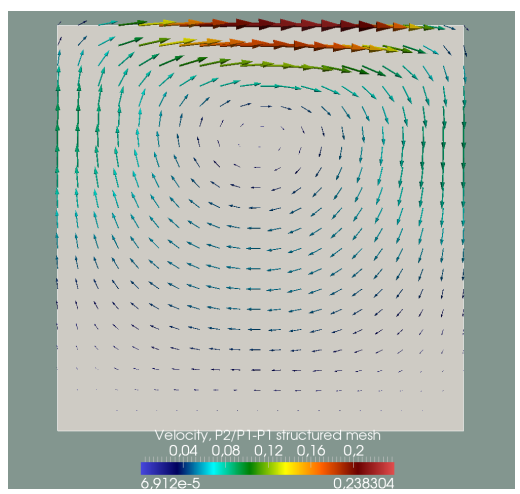

(b) Velocity field.

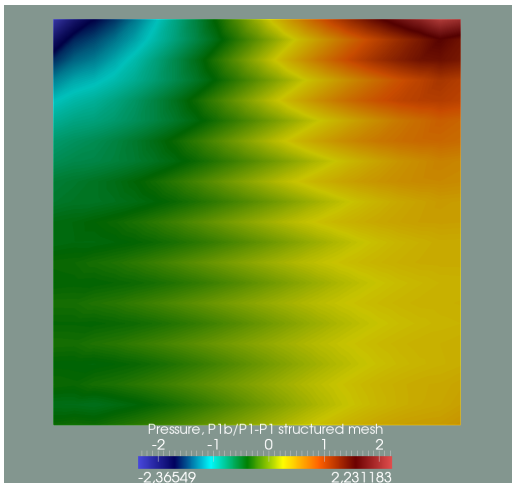

(c) Pressure.

Figure 6: $\left(\mathcal{P}_{1, b}, \mathcal{P}_{1}\right)-\mathcal{P}_{1}$ elements in a structured mesh. 

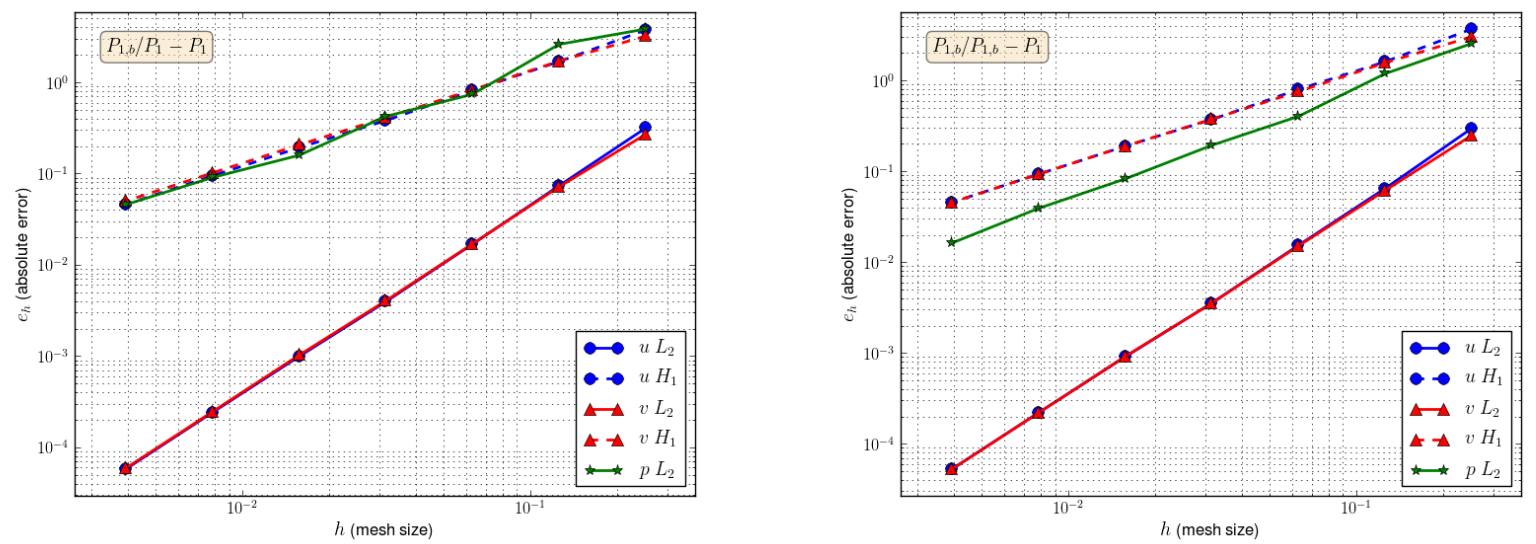

Figure 7: Velocity and pressure errors for $\left(\mathcal{P}_{1, b}, \mathcal{P}_{1}\right)-\mathcal{P}_{1}$ (left) and $\left(\mathcal{P}_{1, b}, \mathcal{P}_{1, b}\right)-\mathcal{P}_{1}$ (right)

(Figure 5c), presents clear non-physical oscillations in the unstructured one (Figure 6c) which has a somewhat "checker-board" structure similar to classical unstable approximations of Stokes problem [GR86]. Hence this experiment confirm that $\left(\mathcal{P}_{1, b}, \mathcal{P}_{1}\right)-\mathcal{P}_{1}$ is stable in meshes which cannot be split by any horizontal segment, as predicted by the $2 D$ numerical analysis developed in Section 3.1.

Test $3\left(\left(\mathcal{P}_{1, b}, \mathcal{P}_{1}\right)-\mathcal{P}_{1}\right.$ and error orders $)$. Thirdly, to give an estimate of the convergence orders, we have considered a $2 \mathrm{D}$ example in $\Omega=(0,1)^{2}$ whose exact solution is:

$$
\begin{aligned}
& u(x, y)=\cos (2 \pi x) \sin (2 \pi y)-\sin (2 \pi y), \quad v(x, y)=-u(y, x), \\
& p(x, y)=2 \pi(\cos (2 \pi y)-\cos (2 \pi x)) .
\end{aligned}
$$

Note that $\partial_{x} u+\partial_{y} v=0,\left.(u, v)\right|_{\partial \Omega}=0$ and $\int_{\Omega} p=0$. The external force $\mathbf{f}$, is calculated so that the momentum equation (1) hold.

The problem has been solved in some unstructured meshes (recall that the theory predicts instability of $\left(\mathcal{P}_{1, b}, \mathcal{P}_{1}\right)-\mathcal{P}_{1}$ in structured meshes), with $h \simeq 2^{-2}, 2^{-3}, \ldots, 2^{-8}$. The absolute errors obtained have been plotted in Figure $7 \mathrm{a}$, while Table 1 shows the values of the convergence orders associated to those errors, which are calculated as $\log \left(e_{h_{2}} / e_{h_{1}}\right) / \log \left(h_{2} / h_{1}\right)$, when $h_{1}<h_{2}$ travel through the different mesh sizes.

Taking into account error estimates (19), we can state the following conclusions:

- For both components of the velocity field, optimal convergence is obtained, that is order $O\left(h^{2}\right)$ in $L^{2}(\Omega)$ and order $O(h)$ in $H^{1}(\Omega)$. For pressure, optimal order $O(h)$ in $L^{2}(\Omega)$ is obtained.

- The results are very similar (almost identical for the velocity components) to the classical mini-element, $\left(\mathcal{P}_{1, b}, \mathcal{P}_{1, b}\right)-\mathcal{P}_{1}$, whose errors are shown in Figure $7 \mathrm{~b}$. It is significant to note that the combination $\left(\mathcal{P}_{1, b}, \mathcal{P}_{1}\right)-\mathcal{P}_{1}$ preserves the error orders while requires a minor number of degrees of freedom than $\left(\mathcal{P}_{1, b}, \mathcal{P}_{1, b}\right)-\mathcal{P}_{1}$ and a smaller computational effort. On the other hand, to approach the Anisotropic Stokes problem (see (AS) above), $\left(\mathcal{P}_{1, b}, \mathcal{P}_{1}\right)-\mathcal{P}_{1}$ is stable in most meshes, while $\left(\mathcal{P}_{1, b}, \mathcal{P}_{1, b}\right)-\mathcal{P}_{1}$ looses its stability (cf. [GR14]).

Test 4 (Post-precessing Algorithm 1). We have used Algorithm 1 (with $r=0.15$ ) for modifying a strongly structured mesh (defined by $16 \times 16$ intervals in the unit square, see Figure $8 \mathrm{a}$ ), arriving 


\begin{tabular}{cccccccc}
\hline & $h$ & $2^{-3}$ & $2^{-4}$ & $2^{-5}$ & $2^{-6}$ & $2^{-7}$ & $2^{-8}$ \\
\hline \multirow{2}{*}{$u$} & $\left\|u-u_{h}\right\|_{L^{2}}$ & 1.099 & 1.709 & 1.952 & 1.876 & 1.988 & 2.019 \\
\cline { 2 - 8 } & $\left\|u-u_{h}\right\|_{H_{0}^{1}}$ & 1.155 & 1.053 & 1.092 & 0.990 & 1.023 & 1.041 \\
\hline \multirow{2}{*}{$v$} & $\left\|v-v_{h}\right\|_{L^{2}}$ & 1.914 & 2.076 & 2.071 & 1.981 & 2.059 & 2.058 \\
\cline { 2 - 8 } & $\left\|v-v_{h}\right\|_{H_{0}^{1}}$ & 0.912 & 1.046 & 1.010 & 0.994 & 1.022 & 1.022 \\
\hline \multirow{2}{*}{$p$} & $\left\|p-p_{h}\right\|_{L^{2}}$ & 0.558 & 1.817 & 0.808 & 1.414 & 0.809 & 0.988 \\
\hline
\end{tabular}

Table 1: Error orders for velocities and pressure $\left(\left(\mathcal{P}_{1, b}, \mathcal{P}_{1}\right)-\mathcal{P}_{1} \mathrm{FE}\right)$

at the unstructured mesh given in Figure 8b. Pressure for a standard cavity-driven test shows instabilities for $\left(\mathcal{P}_{1, b}, \mathcal{P}_{1}\right)-\mathcal{P}_{1}$ in the original structured mesh (Figure 8a) but not in the postprocessed one (Figure $8 \mathrm{~b}$ ).

Test 5 (3D domain, $\left(\mathcal{P}_{1, b}, \mathcal{P}_{1, b}, \mathcal{P}_{1}\right)-\mathcal{P}_{1}$ or $\left(\mathcal{P}_{1, b}, \mathcal{P}_{1}, \mathcal{P}_{1}\right)-\mathcal{P}_{1}$ and cavity test). In the $3 D$ case, we have approximated the solution of the Stokes equations (1)-(3) in the cube domain $\Omega=(0,1)^{3} \subset$ $\mathbb{R}^{3}$, using both:

1. A structured mesh, constructed by FreeFem++ [PHLHM] through the subdivision in three tetrahedrons of each ones of the cubes resulting of the division of $\Omega$ into $32^{3}$ equal rectangular parallelepipeds.

2. a unstructured mesh consisting of 190,968 tetrahedrons, constructed by the mesh generator Gmsh [GR09] and then imported to FreeFem++.

The Dirichlet condition $u(x, y, z)=y(y-1), v(x, y, z)=w(x, y, z)=0$ has been fixed on the top boundary, while $\mathbf{w}=0$ has been fixed on the rest of $\partial \Omega$ (where $\mathbf{w}=(u, v, w)$ denotes the solution). The FE approximations $\left(\mathcal{P}_{1, b}, \mathcal{P}_{1, b}, \mathcal{P}_{1, b}\right)-\mathcal{P}_{1},\left(\mathcal{P}_{1, b}, \mathcal{P}_{1, b}, \mathcal{P}_{1}\right)-\mathcal{P}_{1}$ and $\left(\mathcal{P}_{1, b}, \mathcal{P}_{1}, \mathcal{P}_{1}\right)-\mathcal{P}_{1}$ have been used in each one of the two meshes and the resulting surface plots of the approximated pressure, $p_{h}$, is shown in Figure 9 (structured mesh) and Figure 10 (unstructured one).

Owing to the choice of a color map that highlights the small absolute values of $p_{h}$, spurious oscillations of the pressure are evident for $\left(\mathcal{P}_{1, b}, \mathcal{P}_{1, b}, \mathcal{P}_{1}\right)-\mathcal{P}_{1}$ and $\left(\mathcal{P}_{1, b}, \mathcal{P}_{1}, \mathcal{P}_{1}\right)-\mathcal{P}_{1}$ in the structured mesh (Figures $9 \mathrm{~b}$ and $9 \mathrm{c}$ ), where we have stated their instability. On the other hand, Figures $10 \mathrm{~b}$ and 10c suggest a correct behaviour (excepting some small oscillations on the top of the domain) if the mesh is unstructured, confirming theoretical results in Section 3.2.

Test 6 ( $3 D$ domain, enriching with bubble in the correct direction). In this test it has been considered a mesh $\mathcal{T}_{h}$ of the cubic $\Omega=(0,1)^{3}$, which presents a clear structure only in the $z$-direction. Namely, $\mathcal{T}_{h}$ is $z$-structured while $x$-unstructured and $y$-unstructured.

In concrete, we have considered at the top of the domain, $S$, an unstructured triangulation $\mathcal{T}_{h 2}$ of $S$ (defined with 64 sub-intervals on $\partial S$, i.e. $h \simeq 0.015$ ). Then a $3 D$ mesh, $\mathcal{T}_{h}$, has been constructed, defining it from the extension of $\mathcal{T}_{h 2}$ along 16 layers uniformly distributed in the $z$-direction.

We have programed the lid driven cavity test (as detailed in previous tests) for $\left(\mathcal{P}_{1, b}, \mathcal{P}_{1, b}, \mathcal{P}_{1, b}\right)-\mathcal{P}_{1}$, $\left(\mathcal{P}_{1}, \mathcal{P}_{1}, \mathcal{P}_{1, b}\right)-\mathcal{P}_{1}$ and $\left(\mathcal{P}_{1, b}, \mathcal{P}_{1}, \mathcal{P}_{1}\right)-\mathcal{P}_{1} \mathrm{FE}$ combinations. In the resulting graphics for pressure, shown in Figure 11, it can be seen that the two first cases (Figures 11a and 11b) present a similar correct qualitative behaviour, while the third one (Figure 11c) presents some non physical oscillations (which, in this graphics, are amplified by the selection of the color map). 


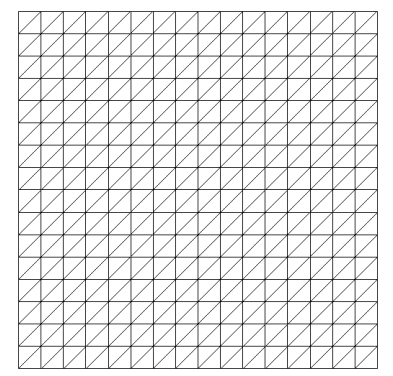

(a) Original structured mesh

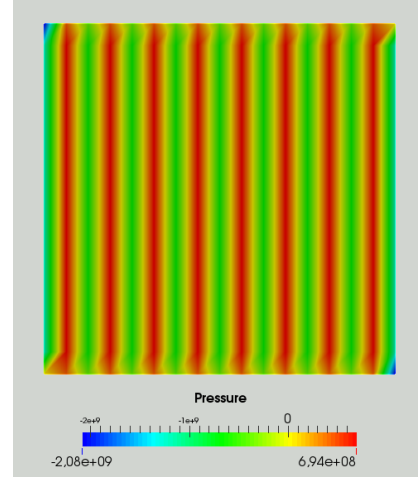

(c) Pressure in structured mesh

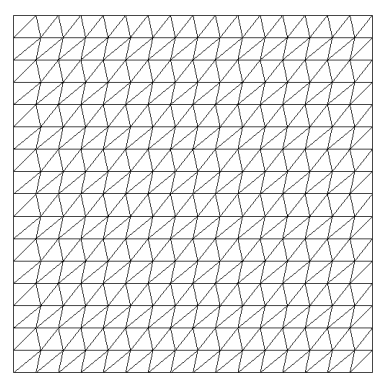

(b) Post-processed unstructured mesh

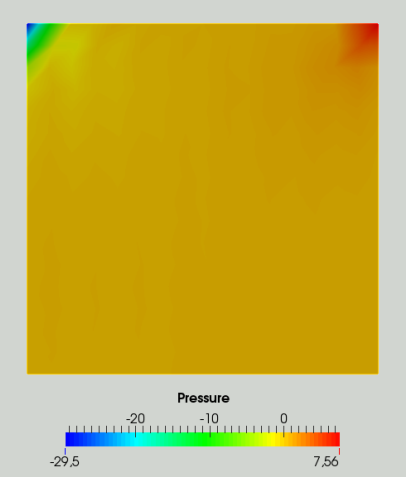

(d) Pressure in post-processed mesh

Figure 8: Test of Algorithm 1 in a structured mesh.

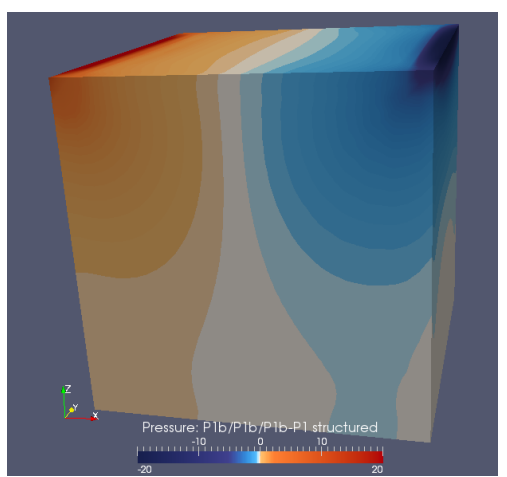

(a) $\left(\mathcal{P}_{1, b}, \mathcal{P}_{1, b}, \mathcal{P}_{1, b}\right)-\mathcal{P}_{1}$

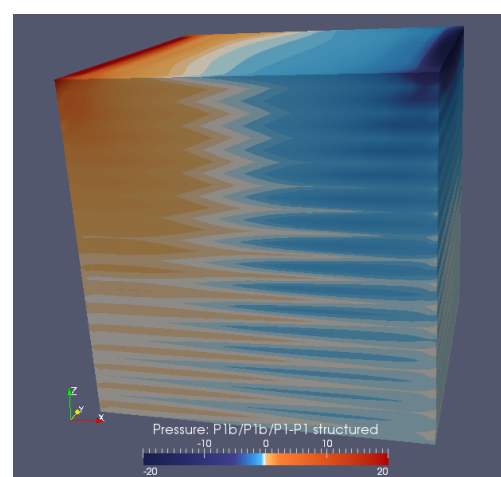

(b) $\left(\mathcal{P}_{1, b}, \mathcal{P}_{1, b}, \mathcal{P}_{1}\right)-\mathcal{P}_{1}$

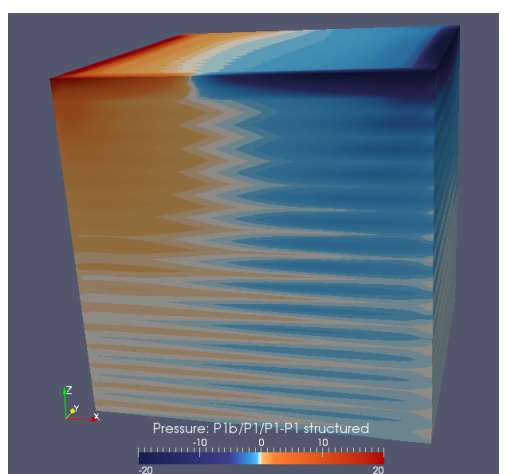

(c) $\left(\mathcal{P}_{1, b}, \mathcal{P}_{1}, \mathcal{P}_{1}\right)-\mathcal{P}_{1}$

Figure 9: Comparison of bubble $3 D \mathrm{FE}$ in a structured mesh. 


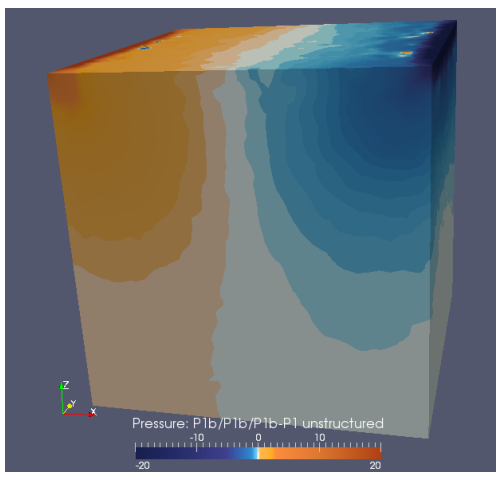

(a) $\left(\mathcal{P}_{1, b}, \mathcal{P}_{1, b}, \mathcal{P}_{1, b}\right)-\mathcal{P}_{1}$

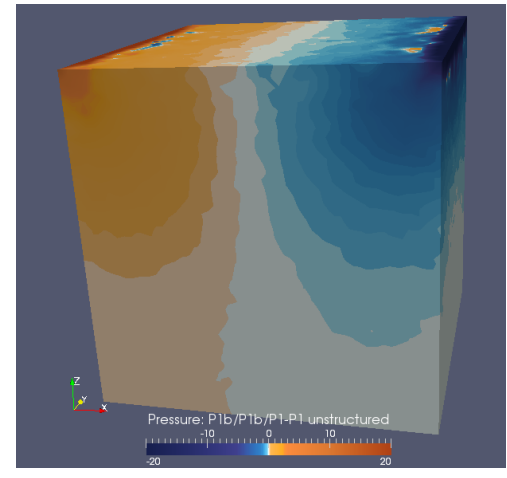

(b) $\left(\mathcal{P}_{1, b}, \mathcal{P}_{1, b}, \mathcal{P}_{1}\right)-\mathcal{P}_{1}$

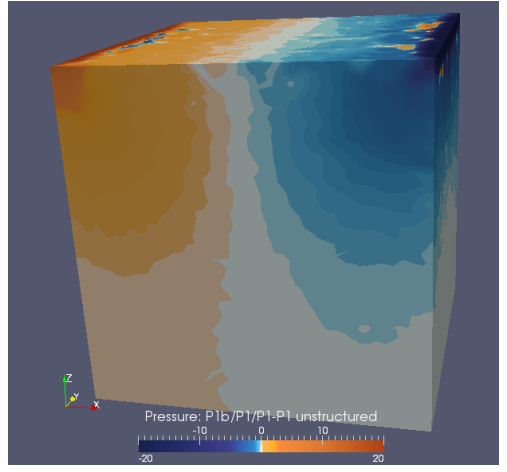

(c) $\left(\mathcal{P}_{1, b}, \mathcal{P}_{1}, \mathcal{P}_{1}\right)-\mathcal{P}_{1}$

Figure 10: Comparison of bubble $3 D \mathrm{FE}$ in an unstructured mesh.

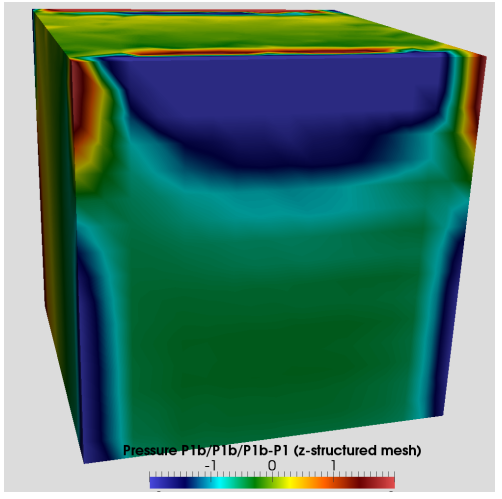

(a) $\left(\mathcal{P}_{1, b}, \mathcal{P}_{1, b}, \mathcal{P}_{1, b}\right)-\mathcal{P}_{1}$

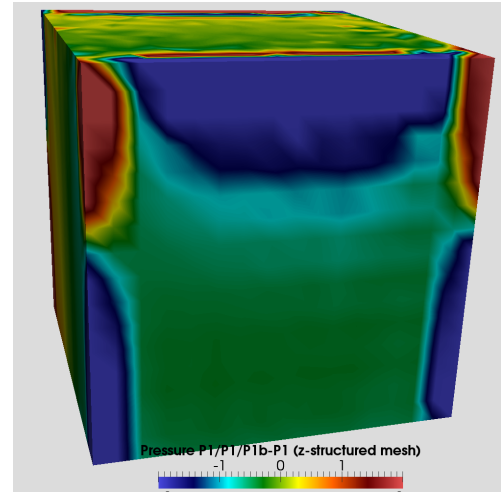

(b) $\left(\mathcal{P}_{1}, \mathcal{P}_{1}, \mathcal{P}_{1, b}\right)-\mathcal{P}_{1}$

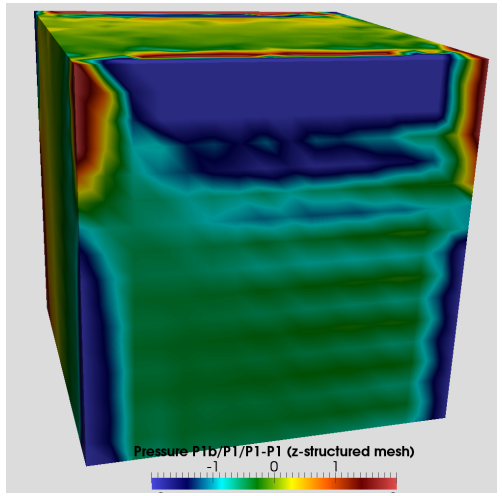

(c) $\left(\mathcal{P}_{1, b}, \mathcal{P}_{1}, \mathcal{P}_{1}\right)-\mathcal{P}_{1}$

Figure 11: Comparison of bubble $3 D$ FE in a $z$-structured mesh.

This behaviour agree with the theory developed in Section 3.2, which suggest the stability of $\left(\mathcal{P}_{1}, \mathcal{P}_{1}, \mathcal{P}_{1, b}\right)-\mathcal{P}_{1}$. Indeed, for this mesh, we can hope that the macro-elements $\widehat{M} \in \widehat{\mathcal{M}}_{h}$, are $x$-unstructured and $y$-unstructured, hence adding bubble functions to the first components of the velocity field is not mandatory. But these macro-elements are $z$-structured and then, for obtaining regularity, it is necessary and sufficient adding bubble functions to the third component. Also due to this fact, we cannot hope stability for $\left(\mathcal{P}_{1, b}, \mathcal{P}_{1}, \mathcal{P}_{1}\right)-\mathcal{P}_{1}$, (see Figure $11 \mathrm{c}$ ).

\section{About stability of $\left(\mathcal{P}_{2}, \mathcal{P}_{1}\right)-\mathcal{P}_{1}$ FE}

\subsection{The $2 \mathrm{D}$ case}

In this section we introduce a $\mathcal{P}_{2}$ continuous space for the approximation of the horizontal component of velocity field,

$$
U_{h}=\left\{u_{h} \in H_{0}^{1}(\Omega) \cap C^{0}(\Omega) /\left.u_{h}\right|_{T} \in \mathcal{P}_{2}, \forall T \in \mathcal{T}_{h}\right\},
$$

while $V_{h}$ and $P_{h}$ are approximated by $\mathcal{P}_{1}$ continuous elements, as defined in (8) and (11).

As in Section 3.1, let us suppose $\mathcal{T}_{h}$ satisfying Assumption 1 and let $\widehat{\mathcal{M}}_{h}$ be the a vertexcentered macro-element partitioning of $\mathcal{T}_{h}$, as in Figure 12. Let $U_{0, M}$ be the space of functions in $H_{0}^{1}(M) \cap C^{0}(\bar{M})$ which are $\mathcal{P}_{2}$ in each element $T \subset M$ and let $V_{0, M}$ and $P_{M}$ as in Section 3.1. In 


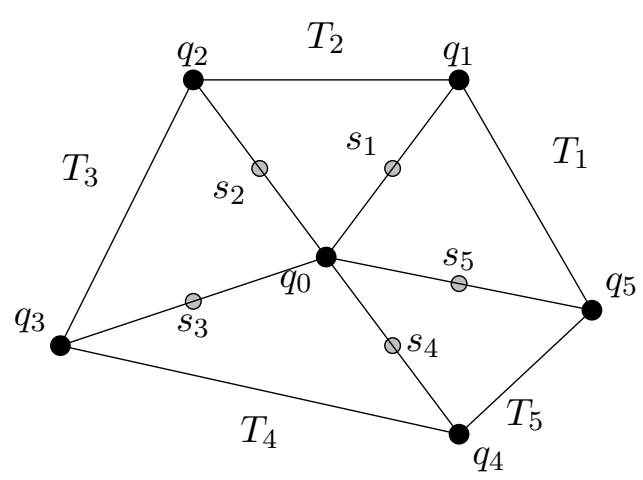

Figure 12: $\left(\mathcal{P}_{2}, \mathcal{P}_{1}\right)-\mathcal{P}_{1}$ macro-element with a unique interior vertex, $q_{0}$, and $\mathrm{n}_{v}=5$.

this framework, Lemma 2 means that a sufficient condition for the discrete inf-sup condition (6) is that $\widehat{\mathcal{M}}_{h}$ satisfies (12), namely $\left(\mathcal{P}_{2}, \mathcal{P}_{1}\right)-\mathcal{P}_{1}$ is regular in every $\widehat{M} \in \widehat{\mathcal{M}}_{h}$. The following result characterizes this condition in function of the number of elements contained in $\widehat{M}$ and the number of vertices which are horizontally aligned with $q_{0}$. See that this case is a little more technical than the similar one (Theorem 3) presented in Section 3.1 (see Remark 8 for further details).

Theorem 9. Let $\widehat{M} \in \widehat{\mathcal{M}}_{h}$ be a macro-element which can be written as union of $\mathrm{n}_{v}$ elements which share exactly one interior common vertex, $q_{0}$.

1. If $\widehat{M}$ is $y$-structured (i.e. there are two vertices horizontally aligned to $\left.q_{0}\right)$, then $\left(\mathcal{P}_{2}, \mathcal{P}_{1}\right)-\mathcal{P}_{1}$ is not regular in $\widehat{M}$, namely condition (12) does not hold.

2. If there is just one vertex in $\widehat{M}$ horizontally aligned with $q_{0}$, then $\left(\mathcal{P}_{2}, \mathcal{P}_{1}\right)-\mathcal{P}_{1}$ is regular in $M$.

3. If there is no vertex horizontally aligned with $q_{0}$ :

(a) If $\mathrm{n}_{v}$ is odd, then $\left(\mathcal{P}_{2}, \mathcal{P}_{1}\right)-\mathcal{P}_{1}$ is regular in $\widehat{M}$.

(b) If $\mathrm{n}_{v}$ is even, then $\left(\mathcal{P}_{2}, \mathcal{P}_{1}\right)-\mathcal{P}_{1}$ is regular in $\widehat{M}$ if and only if the following algebraic condition does not hold:

$$
\sum_{i=1}^{\mathrm{n}_{v}}(-1)^{i} \cot \left(\sigma_{i}\right)\left(\frac{1}{\left|T_{i}\right|}+\frac{1}{\left|T_{i+1}\right|}\right)=0
$$

where $\cot \left(\sigma_{i}\right)=\cos \left(\sigma_{i}\right) / \sin \left(\sigma_{i}\right)$ is the cotangent function and $\sigma_{i}$ are the angles introduced before Definition 6

Remark 6. The case $\left(\mathcal{P}_{1}, \mathcal{P}_{2}\right)-\mathcal{P}_{1}$ is analogue and, for brevity, is not enounced here.

Proof. We split the proof into four steps. 
Step 1. Algebraic characterization of $N_{\widehat{M}}$.

Let $T_{i}, i=1, \ldots, \mathrm{n}_{v}$, be the elements of $\widehat{M}$. If $p_{h} \in N_{\widehat{M}}$, let us define the form functions $\left.p_{h}\right|_{T_{i}}=$ $a_{i}+b_{i} x+c_{i} y$. As in Figure 12, let us denote by $q_{0}$ the only vertex interior to $\widehat{M}, q_{i}, i=1, \ldots \mathrm{n}_{v}$, the vertices in $\partial \widehat{M}$ and $s_{i}, i=1, \ldots, \mathrm{n}_{v}$ the midpoints of the edges in the interior of $\widehat{M}$.

Choosing $v_{h}=0$ and $u_{h} \in U_{0, \widehat{M}}$ defined as $u_{h}=1$ on a midpoint $s_{i}$ and $u_{h}=0$ on all other degrees of freedom of $\widehat{M}\left(s_{j}, j=1, \ldots, \mathrm{n}_{v}, j \neq i\right.$ and $\left.q_{k}, k=0, \ldots, \mathrm{n}_{v}\right)$, then

$$
\begin{aligned}
0=-\int_{\widehat{M}} \nabla \cdot\left(u_{h}, v_{h}\right) p_{h} & =-\int_{\widehat{M}} \partial_{x} u_{h} p_{h}=\int_{\widehat{M}} u_{h} \partial_{x} p_{h}= \\
& =b_{i} \int_{T_{i}} u_{h}+b_{i+1} \int_{T_{i+1}} u_{h}=\frac{\left|T_{j}\right|}{3} b_{j}+\frac{\left|T_{j+1}\right|}{3} b_{j+1},
\end{aligned}
$$

where we have applied the quadrature formula $\int_{T} f=\frac{|T|}{3} \sum_{i=1}^{3} f\left(s_{i}^{T}\right)$, which is exact on $\mathcal{P}_{2}$ if $s_{i}^{T}$ are the midpoints of the edges of $T$. Again, we have identified the index $i=\mathrm{n}_{v}+1$ with $i=1$. Defining $\alpha_{i}=\left|T_{i}\right|$, the following linear system for $b_{i}$ is obtained:

$$
\left(\begin{array}{cccccc}
\alpha_{1} & \alpha_{2} & 0 & \ldots & 0 & 0 \\
0 & \alpha_{2} & \alpha_{3} & \ldots & 0 & 0 \\
\vdots & \vdots & \vdots & \ddots & \vdots & \vdots \\
0 & 0 & 0 & \ldots & \alpha_{\mathrm{n}_{v}-1} & \alpha_{\mathrm{n}_{v}} \\
\alpha_{1} & 0 & 0 & \ldots & 0 & \alpha_{\mathrm{n}_{v}}
\end{array}\right) \cdot\left(\begin{array}{c}
b_{1} \\
b_{2} \\
\vdots \\
b_{\mathrm{n}_{v}-1} \\
b_{\mathrm{n}_{v}}
\end{array}\right)=\left(\begin{array}{c}
0 \\
0 \\
\vdots \\
0 \\
0
\end{array}\right)
$$

A simple calculation shows that, if $b_{1}=-b / \alpha_{1}$ then $b_{2}=b / \alpha_{2}, b_{3}=-b / \alpha_{3}, \ldots, b_{\mathrm{n}_{v}}=(-1)^{\mathrm{n}_{v}} b / \alpha_{\mathrm{n}_{v}}$ and $b_{1}=(-1)^{\mathrm{n}_{v}+1} b / \alpha_{1}$. Therefore, the solution of (32) can be characterized as follows:

$$
\left\{\begin{array}{l}
\text { If } \mathrm{n}_{v} \text { is odd, } \quad b_{i}=0, \quad \forall i=1, \ldots, \mathrm{n}_{v} . \\
\text { If } \mathrm{n}_{v} \text { is even, } \quad b_{i}=\frac{(-1)^{i}}{\alpha_{i}} b, \quad \forall i=1, \ldots, \mathrm{n}_{v}, \quad \forall b \in \mathbb{R} .
\end{array}\right.
$$

Taking $u_{h}=1$ in $q_{0}, u_{h}=0$ on all other degrees of freedom in $\widehat{M}$ and $v_{h}=0$ does not provide any new information, because the midpoint quadrature formula means that $\int_{T_{i}} u_{h}=0$ for all $i$.

Finally let us choose $u_{h}=0$ and $v_{h} \in V_{0, \widehat{M}}$ defined as $v_{h}=1$ on the interior vertex, $q_{0}$, and of course $v_{h}=0$ on all vertices on $\partial \widehat{M}$. Then $0=\left(v_{h}, \partial_{y} p_{h}\right)=\sum_{i=1}^{\mathrm{n}_{v}} c_{i} \int_{T_{i}} v_{h}=\sum_{i=1}^{\mathrm{n}_{v}} c_{i}\left|T_{i}\right| / 3$ hence

$$
\sum_{i=1}^{\mathrm{n}_{v}} \alpha_{i} c_{i}=0
$$

Now assuming, without loss of generality, $q_{0}=(0,0)$ and imposing the continuity of $p_{h}$ on $q_{0}$, it is straightforward that

$$
a_{1}=\ldots=a_{\mathrm{n}_{v}}=: a .
$$

If $q_{i}=\left(q_{i}^{x}, q_{i}^{y}\right) \in \partial \widehat{M}, i=1, \ldots, \mathrm{n}_{v}$, being $q_{0}$ and $q_{i}$ the common vertices to $T_{i}$ and $T_{i+1}$ (Figure 12), continuity of $p_{h}$ in $q_{i}$ means:

$$
q_{i}^{y}\left(c_{i+1}-c_{i}\right)=q_{i}^{x}\left(b_{i+1}-b_{i}\right), \quad \forall i=1, \ldots, \mathrm{n}_{v} .
$$


Step 2. Proof of case 2.

If there exists a unique vertex on $\partial \widehat{M}$, without loss of generality it can be named $q_{1}$, such that $q_{1}^{y}=0$ (that is, $q_{1}$ is horizontally aligned with $q_{0}$ ), the equation for $i=1$ in (35) means $b_{2}-b_{1}=0$ (note that $q_{1}^{x} \neq 0$ ). According to (33), this does not give any new information in the case of $\mathrm{n}_{v}$ odd (in which $b_{i}=0$ for all $i$ ) but in the even case, we obtain

$$
\left(\frac{(-1)^{2}}{\alpha_{2}}-\frac{(-1)^{1}}{\alpha_{1}}\right) b=0
$$

that is $b=0$ and then $b_{i}=0$ for $i=1, \ldots, \mathrm{n}_{v}$. Now from (35) there exists $c \in \mathbb{R}$ such that $c_{2}=c_{3}=\ldots=c_{\mathrm{n}_{v}}=c_{1}=c$. Then, from (34) $c=0$ (and $c_{i}=0$ for all $i$ ), therefore $p_{h}=a$ and the macro-element condition (12) holds.

Step 3. Proof of case 3.

Now let us suppose that there is not any vertex horizontally aligned with $q_{0}=(0,0)$, that is $q_{i}^{y} \neq 0$ for all $i=1, \ldots, \mathrm{n}_{v}$. According to (33), the linear equations (35) can be written as

$$
\begin{aligned}
q_{i}^{y}\left(c_{i+1}-c_{i}\right) & =q_{i}^{x}\left(\frac{(-1)^{i+1}}{\alpha_{i+1}}-\frac{(-1)^{i}}{\alpha_{i}}\right) b \\
& =q_{i}^{x}(-1)^{i+1}\left(\frac{1}{\alpha_{i+1}}+\frac{1}{\alpha_{i}}\right) b, \quad \forall i=1, \ldots, \mathrm{n}_{v},
\end{aligned}
$$

with $b=0$ if $\mathrm{n}_{v}$ is odd. We can divide by $q_{i}^{y}$, obtaining

$$
\left(c_{i+1}-c_{i}\right)+(-1)^{i} \widehat{m}_{i} \widehat{\alpha}_{i} b=0, \quad \forall i=1, \ldots, \mathrm{n}_{v},
$$

where $\widehat{m}_{i}=q_{i}^{x} / q_{i}^{y}$ is the inverse of the slope of the segment $\overline{q_{0} q_{i}}$, hence $\widehat{m}_{i}=\cos \left(\sigma_{i}\right) / \sin \left(\sigma_{i}\right)=$ $\cot \left(\sigma_{i}\right)$, and $\widehat{\alpha}_{i}=1 / \alpha_{i}+1 / \alpha_{i+1}=1 /\left|T_{i}\right|+1 /\left|T_{i+1}\right|$. Therefore,

$$
\widehat{m}_{i} \widehat{\alpha}_{i}=\cot \left(\sigma_{i}\right)\left(\frac{1}{\left|T_{i}\right|}+\frac{1}{\left|T_{i+1}\right|}\right) \text {. }
$$

If $\mathrm{n}_{v}$ is odd (case 3.a), $b=0$ and (34) and (36) imply $c_{i}=0$ for all $i=1, \ldots, \mathrm{n}_{v}$, concluding that $p_{h}=a$ and (12) holds.

If $\mathrm{n}_{v}$ is even (case 3.b), let us write (36) and (34) as the algebraic system:

$$
\left(\begin{array}{ccccccc}
-1 & 1 & 0 & \ldots & 0 & 0 & -\widehat{m}_{1} \widehat{\alpha}_{1} \\
0 & -1 & 1 & \ldots & 0 & 0 & \widehat{m}_{2} \widehat{\alpha}_{2} \\
\vdots & \vdots & \vdots & \ddots & \vdots & \vdots & \vdots \\
0 & 0 & 0 & \ldots & -1 & 1 & -\widehat{m}_{\mathrm{n}_{v}-1} \widehat{\alpha}_{\mathrm{n}_{v}-1} \\
1 & 0 & 0 & \ldots & 0 & -1 & \widehat{m}_{\mathrm{n}_{v}} \widehat{\alpha}_{\mathrm{n}_{v}} \\
\alpha_{1} & \alpha_{2} & \alpha_{3} & \ldots & \alpha_{\mathrm{n}_{v}-1} & \alpha_{\mathrm{n}_{v}} & 0
\end{array}\right) \cdot\left(\begin{array}{c}
c_{1} \\
c_{2} \\
\vdots \\
c_{\mathrm{n}_{v}-1} \\
c_{\mathrm{n}_{v}} \\
b
\end{array}\right)=\left(\begin{array}{c}
0 \\
0 \\
\vdots \\
0 \\
0 \\
0
\end{array}\right) .
$$

Adding the first $\mathrm{n}_{v}$ rows of this system, we obtain the equation $S b=0$, where

$$
S=\sum_{i=1}^{\mathrm{n}_{v}}(-1)^{i} \widehat{m}_{i} \widehat{\alpha}_{i}=\sum_{i=1}^{\mathrm{n}_{v}}(-1)^{i} \cot \left(\sigma_{i}\right)\left(\frac{1}{\left|T_{i}\right|}+\frac{1}{\left|T_{i+1}\right|}\right) .
$$

If we assume $S \neq 0$, then $b=0$ and the first $\mathrm{n}_{v}$ equations of (37) can be written as

$$
-c_{1}+c_{2}=0, \quad-c_{2}+c_{3}=0, \ldots, \quad-c_{\mathrm{n}_{v}}+c_{1}=0,
$$


hence $c_{1}=c_{2}=\cdots=c_{\mathrm{n}_{v}} \equiv c \in \mathbb{R}$. But then, last equation of (37) means c $\sum_{i=1}^{\mathrm{n}_{v}} \alpha_{i}=0$, which implies $c=0$. In short, if $S \neq 0$ the only solution of the system is $c_{1}=\cdots=c_{\mathrm{n}_{v}}=b=0$ and therefore $p_{h}=a$.

Reciprocally, if $S=0$ the first $\mathrm{n}_{v}$ rows of $A$ are linearly dependent and the system (37) has got non trivial solutions $\left(c_{1}, \ldots, c_{\mathrm{n}_{v}}, b\right) \neq 0$. For those values, any pressure defined by

$$
\left.p_{h}\right|_{T_{i}}=a+\frac{(-1)^{i}}{\alpha_{i}} b x+c_{i} y
$$

satisfies (32)-(35), therefore it is a non-constant element of $N_{\widehat{M}}$ and (12) does not hold.

Step 4. Proof of case 1.

Finally, we show that if there exist two vertices $q_{j}$ and $q_{k}$ on $\partial \widehat{M}$ horizontally aligned with $q_{0}$ $\left(1 \leq j<k \leq \mathrm{n}_{v}\right)$ then $\widehat{M}$ is not regular. Without loss of generality, renumbering the vertices if necessary, we can suppose $1<j<k=\mathrm{n}_{v}$. Then equations $j$ and $\mathrm{n}_{v}$ in (35) imply respectively $b_{j+1}=b_{j}$ and $b_{1}=b_{\mathrm{n}_{v}}$, which (using (33)) yields $b=0$ (in both cases) and then $b_{i}=0$ for all $i=1, \ldots, \mathrm{n}_{v}$.

Taking this into account in the rest of equations of (35), we have

$$
c_{2}-c_{1}=0, \ldots, c_{j}-c_{j-1}=0 \quad \text { and } \quad c_{j+2}-c_{j+1}=0, \ldots, c_{\mathrm{n}_{v}}-c_{\mathrm{n}_{v}-1}=0
$$

and then, there exists $c$ and $\widetilde{c} \in \mathbb{R}$, such that

$$
c_{1}=c_{2}=\cdots=c_{j} \equiv c \quad \text { and } \quad c_{j+1}=c_{j+2}=\cdots=c_{\mathrm{n}_{v}} \equiv \widetilde{c} .
$$

This fact and condition (34) imply

$$
\widetilde{c}=\frac{-\sum_{i=1}^{j} \alpha_{i}}{\sum_{i=j+1}^{\mathrm{n}_{v}} \alpha_{i}} \cdot c .
$$

Note that $\sum_{i=1}^{j} \alpha_{i}$ and $\sum_{i=j+1}^{\mathrm{n}_{v}} \alpha_{i}$ are the measures of the two macro-elements splitting $\widehat{M}$. Therefore, it is clear that any pressure with $\left.p_{h}\right|_{T_{i}}=c$ for $1 \leq i \leq j$ and $\left.p_{h}\right|_{T_{i}}=\widetilde{c}$ for $j+1 \leq i \leq \mathrm{n}_{v}$, with $\widetilde{c}$ given by (38), is a non constant element of $N_{\widehat{M}}$ and then (12) does not hold.

Remark 7. Like in the case of $\left(\mathcal{P}_{1, b}, \mathcal{P}_{1}\right)-\mathcal{P}_{1}$ given in Section 3.1, the ideas of Theorem 9 can be applied to provide counterexamples showing that $\left(\mathcal{P}_{2}, \mathcal{P}_{1}\right)-\mathcal{P}_{1}$ is not stable in some families of strong structured meshes. In fact, the same example given in Remark 3 is valid in the current context.

Remark 8. According to Theorem 9, there are two situations where $\left(\mathcal{P}_{2}, \mathcal{P}_{1}\right)-\mathcal{P}_{1}$ may be singular in a macro-element with one only interior vertex $q_{0}$ :

(a) either there exist two vertices horizontally aligned with $q_{0}$,

(b) or $\mathrm{n}_{v}$ is even and the algebraic condition (31) holds.

The case (a), which is illustrated in Figure 13a, is coincident to the singularity condition studied for $\left(\mathcal{P}_{1, b}, \mathcal{P}_{1}\right)-\mathcal{P}_{1}$ in Section 3 , but the case $(b)$ is specific of $\left(\mathcal{P}_{2}, \mathcal{P}_{1}\right)-\mathcal{P}_{1}$ and does not appears in the $\left(\mathcal{P}_{1, b}, \mathcal{P}_{1}\right)-\mathcal{P}_{1}$ context. In this sense, the regularity of $\left(\mathcal{P}_{2}, \mathcal{P}_{1}\right)-\mathcal{P}_{1}$ is more restrictive than the regularity of $\left(\mathcal{P}_{1, b}, \mathcal{P}_{1}\right)-\mathcal{P}_{1}$.

The case $(a)$ is specially relevant because constitutes the basic brick in many vertical structured meshes, like the one in Figure 3 (used in the counterexample presented in Remark 7). Also this is the kind of mesh used in the numerical experiment that produced the instability given in Figure 15b. 


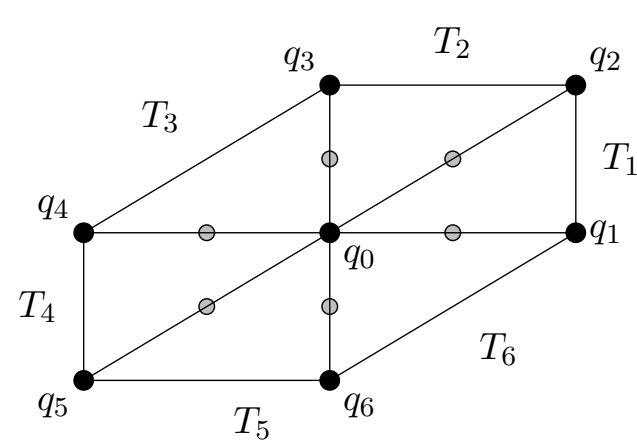

(a) Two vertices horizontally aligned with $q_{0}$.

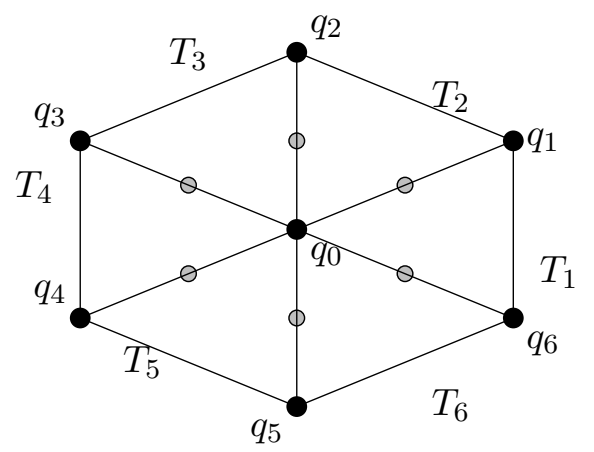

(b) No vertex horizontally aligned, but $n$ is even and (31) holds.

Figure 13: Two macro-elements where $\left(\mathcal{P}_{2}, \mathcal{P}_{1}\right)-\mathcal{P}_{1}$ is not regular.

About condition (b), in practice it is difficult to find random macro-elements of this kind, i.e. macro-elements for which $\mathrm{n}_{v}$ is even, no vertex is horizontally aligned with $q_{0}$ and (31) is satisfied. One of these cases (of what can be called "weakly $y$-structured macro-elements") is presented in Figure 13b. Indeed, assuming $\left|T_{i}\right|=\left|T_{i+1}\right|$ for $i=1, \ldots, 6$, then $\cot \left(\sigma_{i}\right)=1$ for $i=1,4$, $\cot \left(\sigma_{i}\right)=-1$ for $i=3,6$ and $\cot \left(\sigma_{i}\right)=0$ for $i=2,5$, hence (31) holds.

But notice that, in the case of the macro-element in Figure 13b, the continuity of the cotangent function implies that slight modifications of the triangles, conduce to elements where (31) is not satisfied, i.e. regular macro-elements. In any case, for discrete inf-sup condition (6), equation (31) must be uniformly satisfied, i.e. (6) does not hold (with constant independent on $h$ ) if the left hand side in equation (31) tends to zero.

On the other hand, it is easy to find macro-element families where $\left(\mathcal{P}_{2}, \mathcal{P}_{1}\right)-\mathcal{P}_{1}$ is regular. For example, in the case $\mathrm{n}_{v}=4$, if each vertex $q_{i}, i=1, \ldots, 4$ is in a different quadrant (Figure 14a) then the singularity condition (31) does not hold. In effect, if we denote $Q_{i}$ the $i$-th quadrant and suppose $q_{i} \in Q_{i}$ for all $i=1, \ldots, 4$ then $\cot \left(\sigma_{1}\right)>0, \cot \left(\sigma_{2}\right)<0, \cot \left(\sigma_{3}\right)>0, \cot \left(\sigma_{4}\right)<0$ and then

$$
\sum_{i=1}^{4}(-1)^{i} \cot \left(\sigma_{i}\right)\left(\frac{1}{\left|T_{i}\right|}+\frac{1}{\left|T_{i+1}\right|}\right)<0 .
$$

In the case of $\mathrm{n}_{v}=4$ and two vertices in the same quadrant as in Figure 14b, the macro-elements are regular in most cases. For example, let us consider, for simplicity, $\left|T_{i}\right|=1$ for all $i=1, \ldots, 4$. Since we are taking $\sigma_{i}<\sigma_{i+1}$ for $i=1, \ldots, 3$ and the cotangent function is decreasing, if we choose $q_{1}, q_{2} \in Q_{1}$ then $-\cot \left(\sigma_{1}\right)+\cot \left(\sigma_{2}\right):=s_{1,2}<0$. Moreover:

- If $q_{3} \in Q_{2}$ and $q_{4} \in Q_{3}$, then $\cot \left(\sigma_{3}\right)<0$ and $\cot \left(\sigma_{4}\right)>0$. Therefore $-\cot \left(\sigma_{3}\right)+\cot \left(\sigma_{4}\right):=$ $s_{3,4}>0$ and for each angle $\sigma_{3} \in Q_{2}$ there is a unique angle $\sigma_{4} \in Q_{4}$ such that $s_{1,2}+s_{3,4}=0$. In all other cases the singularity condition (31) does not hold.

- If $q_{3} \in Q_{2}$ and $q_{4} \in Q_{4}$, then $\cot \left(\sigma_{3}\right)<0$ and $\cot \left(\sigma_{4}\right)<0$. Therefore $s_{1,2}+\cot \left(\sigma_{4}\right)<0$ and there is a unique $\sigma_{3} \in Q_{3}$ such that (31) holds.

- If $q_{3} \in Q_{3}$ and $q_{4} \in Q_{4}$ (Figure 14b), then $\cot \left(\sigma_{3}\right)>0$ and $\cot \left(\sigma_{4}\right)<0$. Therefore (31) does not hold. 


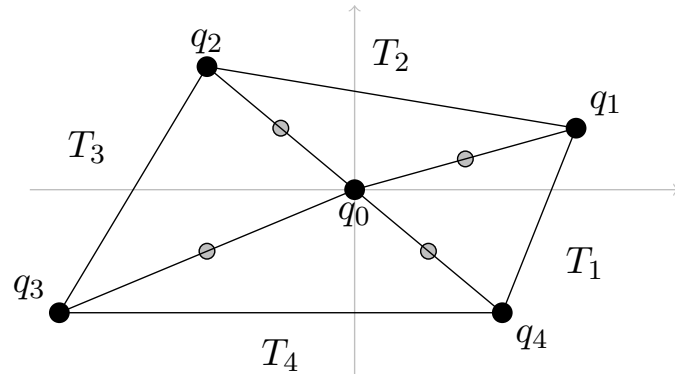

(a) Each boundary vertex is in a different quadrant.

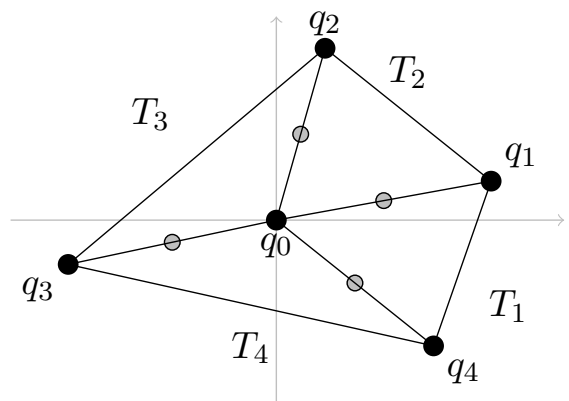

(b) Equal size elements with $q_{1}, q_{2}$ in first quadrant, $q_{3}$ in third and $q_{4}$ in fourth one.

Figure 14: Two $\left(\mathcal{P}_{2}, \mathcal{P}_{1}\right)-\mathcal{P}_{1}$ Stokes-regular macro-elements with $n=4$

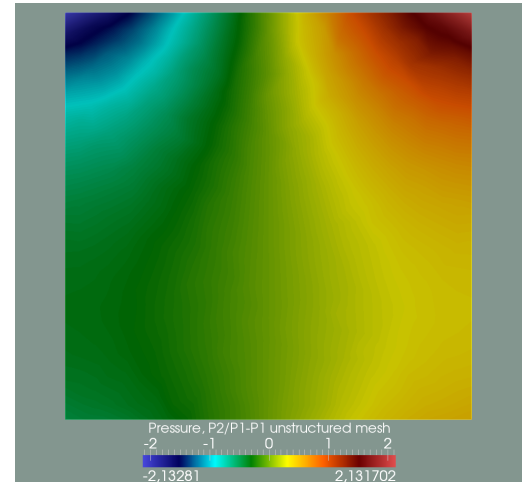

(a) $p_{h}$ in unstructured mesh.

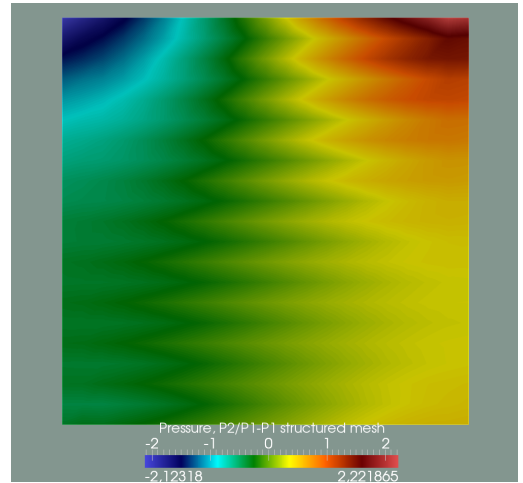

(b) $p_{h}$ in a structured mesh.

Figure 15: $\left(\mathcal{P}_{2}, \mathcal{P}_{1}\right)-\mathcal{P}_{1}$ pressure in unstructured and structured meshes.

Remark 9. Arguing as in Theorem 4, the local result of Theorem 9 can be extended to a global result regarding inf-sup condition (6) in uniformly unstructured meshes. Anyway, as commented in Remark 8, this case is more complicated because it depends also on validating uniformly condition (31). Hence, in principle, the number of meshes where $\left(\mathcal{P}_{2}, \mathcal{P}_{1}\right)-\mathcal{P}_{1}$ is stable is slightly less than in the $\left(\mathcal{P}_{1, b}, \mathcal{P}_{1}\right)-\mathcal{P}_{1}$ case. For ensuring stability, a generalization of Algorithm 1 may be applied. In this case, the algorithm shall be more complex due to the additional condition (31). But in any case, in all our numerical experiments in random (unstructured) meshes, results have been satisfactory without any kind of mesh post-processing. See next section for details.

\subsection{Numerical simulations}

Test $7\left(\left(\mathcal{P}_{2}, \mathcal{P}_{1}\right)-\mathcal{P}_{1}\right.$ and cavity test $)$. Firstly, the cavity test which is described in Test 2 in Section 3 has been repeated for $\left(\mathcal{P}_{2}, \mathcal{P}_{1}\right)-\mathcal{P}_{1}$ elements, using the same unstructured and structured meshes (cf. Figures 5a and 6a). The results obtained, which can be seen in Figure 15, show correct results for the pressure in unstructured meshes (cf. Figure 15a), but spurious pressure modes appear in structured ones (cf. Figure 15b), as expected from the theoretical results given in Section 4.1 (see Remark 8).

Test 8 (Non-uniformly unstructured mesh). In order to present a numerical evidence of the 


\begin{tabular}{cccccc} 
& $\mathcal{T}_{h_{1}}$ & $\mathcal{T}_{h_{2}}$ & $\mathcal{T}_{h_{3}}$ & $\mathcal{T}_{h_{4}}$ & $\mathcal{T}_{h_{5}}$ \\
\hline$h$ & $1 / 3$ & $1 / 7$ & $1 / 15$ & $1 / 31$ & $1 / 63$ \\
\hline$\beta_{h}$ & 0.19384 & 0.096089 & 0.048363 & 0.023503 & 0.0086823 \\
\hline
\end{tabular}

Table 2: Vanishing discrete inf-sup constants on a non-uniformly unstructured mesh family
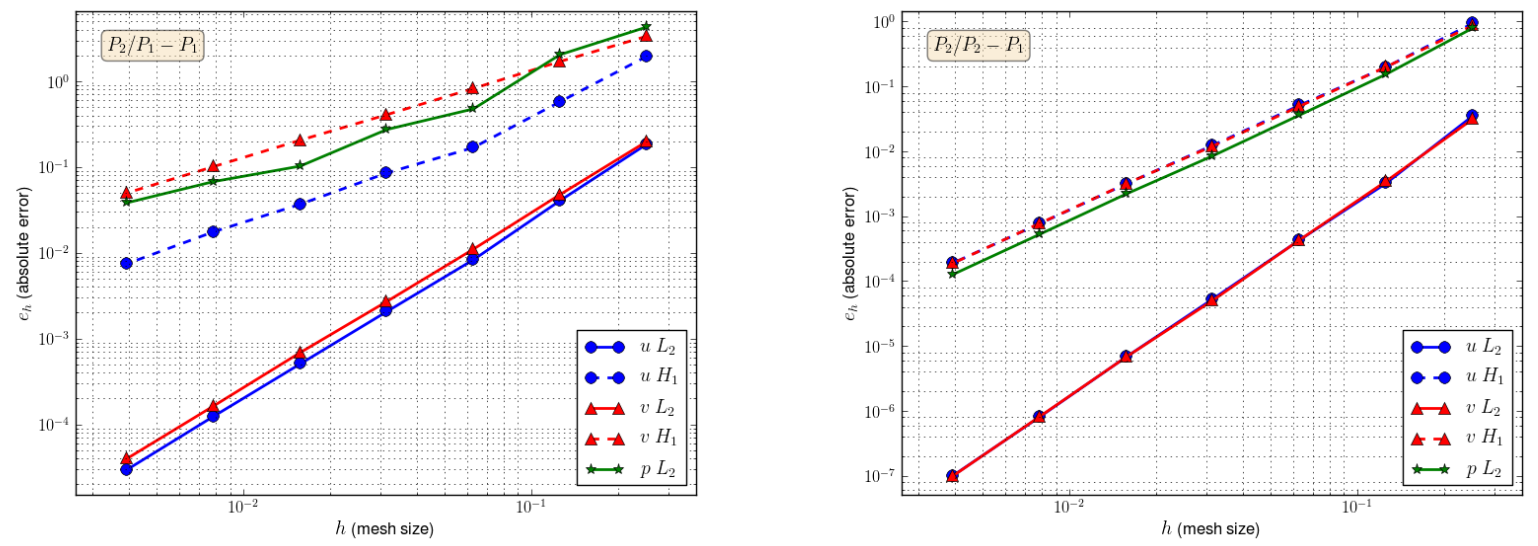

Figure 16: Velocity and pressure errors for $\left(\mathcal{P}_{2}, \mathcal{P}_{1}\right)-\mathcal{P}_{1}$ (left) and $\left(\mathcal{P}_{2}, \mathcal{P}_{2}\right)-\mathcal{P}_{1}$ (right)

necessity of uniform unstructured meshes for stability, we have developed a numerical experiment where the behaviour of $\left(\mathcal{P}_{2}, \mathcal{P}_{1}\right)-\mathcal{P}_{1}$ elements is tested in a non uniformly unstructured mesh family which "converge to a structured mesh" when $h \rightarrow 0$.

Specifically we considered five meshes, $\mathcal{T}_{h_{i}}, i=1, \ldots, 5$, where the finer mesh, $\mathcal{T}_{h_{5}}$, is defined as in Figure 2, with 63 intervals on the top boundary (thus $h_{5} \simeq 1 / 63$ ). About the other coarser four meshes, they are defined similarly (with $3,7,15$ and 31 intervals on the top boundary, respectively) but a random displacement is added to the $x$-coordinate of its vertices, breaking their structure. The amplitude of this displacement decreases $\left(0.4,0.2,0.1,0.05\right.$, respectively), so that $\mathcal{T}_{h_{4}}$ is very similar to $\mathcal{T}_{h_{5}}$. This way, we have a sequence of meshes, $\mathcal{T}_{h_{1}}, \ldots, \mathcal{T}_{h_{4}}$, which are (non uniformly) $y$-unstructured and whose "limit", $\mathcal{T}_{h_{5}}$, is a "weakly $y$-structured" mesh (see Remark 8).

Then we have developed a program which computes estimates of the constants, $\beta_{h}$ related to the discrete inf-sup condition (6) for this mesh family and $\left(\mathcal{P}_{2}, \mathcal{P}_{1}\right)-\mathcal{P}_{1}$ FE. This program is based on ASCoT [AR09], a Python module built on top of the FEniCS framework [LMW ${ }^{+} 12$ ] with the purpose of facilitating the estimation of Brezzi inf-sup constants. Results (see Table 2) suggest that $\beta_{h} \rightarrow 0$ depends on $h$, vanishing when mesh structure appears, therefore discrete inf-sup condition is not satisfied.

Test $9\left(\left(\mathcal{P}_{2}, \mathcal{P}_{1}\right)-\mathcal{P}_{1}\right.$ and error orders $)$. The experiment described in Test 3 has been reproduced for $\left(\mathcal{P}_{2}, \mathcal{P}_{1}\right)-\mathcal{P}_{1}$. Figure 16a shows the absolute errors and orders obtained. The following conclusions can be summarized:

1. For both components of the velocity, order $O\left(h^{2}\right)$ in $L^{2}(\Omega)$ and order $O(h)$ in $H^{1}(\Omega)$ are obtained, while order $O(h)$ for pressure is suggested.

2. Optimal order $O\left(h^{3}\right)$ in $L^{2}(\Omega)$ and $O\left(h^{2}\right)$ in $H^{1}(\Omega)$ is not reached for $u$ (although it is approximated in $\mathcal{P}_{2}$ ), due to the effect of approximating $v$ in $\mathcal{P}_{1}$. Therefore, a loss of accurate 


\begin{tabular}{rccccccc}
\hline & $h$ & $2^{-3}$ & $2^{-4}$ & $2^{-5}$ & $2^{-6}$ & $2^{-7}$ & $2^{-8}$ \\
\hline \multirow{2}{*}{$u$} & $\left\|u-u_{h}\right\|_{L^{2}}$ & 2.185 & 2.291 & 2.008 & 2.029 & 2.031 & 2.066 \\
\cline { 2 - 8 } & $\left\|u-u_{h}\right\|_{H_{0}^{1}}$ & 1.758 & 1.774 & 0.986 & 1.212 & 1.067 & 1.237 \\
\hline \multirow{2}{*}{$v$} & $\left\|v-v_{h}\right\|_{L^{2}}$ & 2.056 & 2.116 & 2.028 & 1.980 & 2.068 & 2.040 \\
\cline { 2 - 8 } & $\left\|v-v_{h}\right\|_{H_{0}^{1}}$ & 0.977 & 1.044 & 1.017 & 0.990 & 1.022 & 1.019 \\
\hline \multirow{2}{*}{$p$} & $\left\|p-p_{h}\right\|_{L^{2}}$ & 1.070 & 2.101 & 0.798 & 1.419 & 0.599 & 0.820 \\
\hline
\end{tabular}

Table 3: Error orders for velocities and pressure $\left(\left(\mathcal{P}_{2}, \mathcal{P}_{1}\right)-\mathcal{P}_{1} \mathrm{FE}\right)$

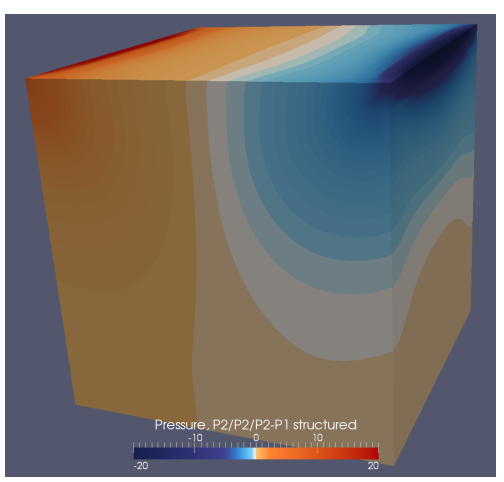

(a) $\left(\mathcal{P}_{2}, \mathcal{P}_{2}, \mathcal{P}_{2}\right)-\mathcal{P}_{1}$

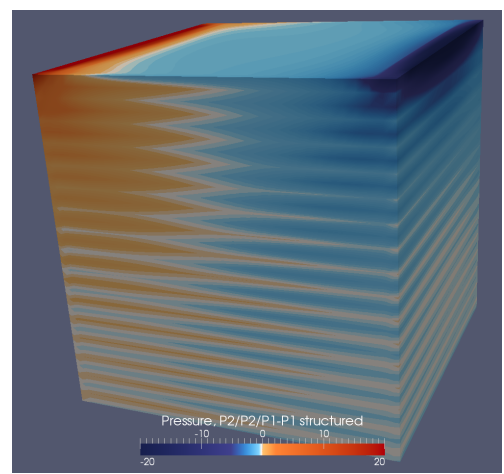

(b) $\left(\mathcal{P}_{2}, \mathcal{P}_{2}, \mathcal{P}_{1}\right)-\mathcal{P}_{1}$

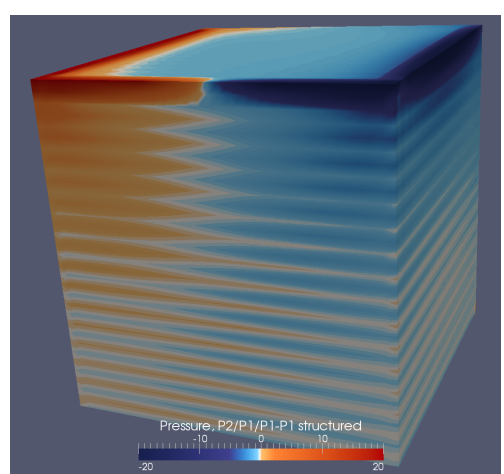

(c) $\left(\mathcal{P}_{2}, \mathcal{P}_{1}, \mathcal{P}_{1}\right)-\mathcal{P}_{1}$

Figure 17: Comparing the pressure in a $3 D$ structured mesh.

is produced passing from the classical Taylor-Hood element $\left(\mathcal{P}_{2}, \mathcal{P}_{2}\right)-\mathcal{P}_{1}$ (Figure 16b) to $\left(\mathcal{P}_{2}, \mathcal{P}_{1}\right)-\mathcal{P}_{1}$ (Figure 16a). In spite of it, this latter element $\left(\mathcal{P}_{2}, \mathcal{P}_{1}\right)-\mathcal{P}_{1}$ is more stable than $\left(\mathcal{P}_{2}, \mathcal{P}_{2}\right)-\mathcal{P}_{1}$ in degenerated anisotropic problems, as is shown in [GR14].

Test 10 (3D domain, $\left(\mathcal{P}_{2}, \mathcal{P}_{2}, \mathcal{P}_{1}\right)-\mathcal{P}_{1}$ or $\left(\mathcal{P}_{2}, \mathcal{P}_{1}, \mathcal{P}_{1}\right)-\mathcal{P}_{1}$ and cavity tests). Finally, we have repeated the $3 D$ experiment which was described in Test 2 , using both structured and unstructured meshes, but replacing the bubble by $\mathcal{P}_{2}$.

Owing to the choice of a color map that amplifies the variations around small values of pressure, spurious oscillations of the $p_{h}$ are evident in the structured mesh for $\left(\mathcal{P}_{2}, \mathcal{P}_{2}, \mathcal{P}_{1}\right)-\mathcal{P}_{1}$ and $\left(\mathcal{P}_{2}, \mathcal{P}_{1}, \mathcal{P}_{1}\right)-\mathcal{P}_{1}$ (Figures $17 \mathrm{~b}$ and $\left.17 \mathrm{c}\right)$

\section{$5 \quad$ Instability of $\left(\mathcal{Q}_{2}, \mathcal{Q}_{1}\right)-\mathcal{Q}_{1}$ in structured meshes}

In this section we present a counterexample showing that the combination $\left(\mathcal{Q}_{2}, \mathcal{Q}_{1}\right)-\mathcal{Q}_{1}$ is not stable in structured quadrangular macro-elements. As above, this result can be extended to global quadrangular meshes.

Let us consider a $2 D$ quadrangular structured mesh $\mathcal{T}_{h}$. Let $M$ be a macro-element as in Figure 19, i.e. $M$ is union of four rectangular elements defined by 9 vertices, which are denoted as $q_{i j}(i, j=1, \ldots, 3)$ and constitute the $\mathcal{Q}_{1}$ degrees of freedom. The rest of the $\mathcal{Q}_{2}$ degrees of freedom are located at the midpoints of the edges and at the center of the quadrangles. Without 


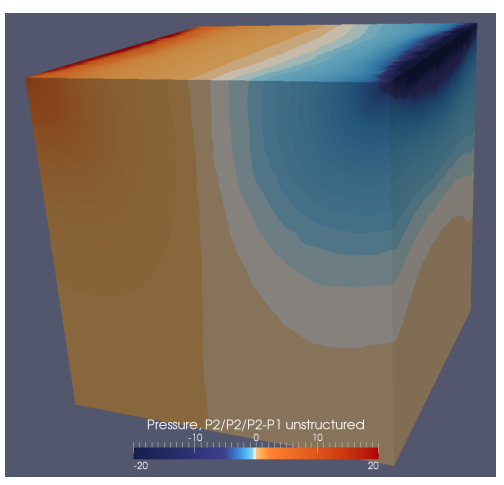

(a) $\left(\mathcal{P}_{2}, \mathcal{P}_{2}, \mathcal{P}_{2}\right)-\mathcal{P}_{1}$

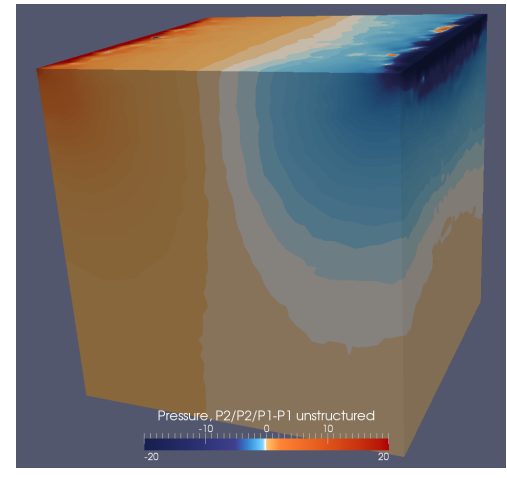

(b) $\left(\mathcal{P}_{2}, \mathcal{P}_{2}, \mathcal{P}_{1}\right)-\mathcal{P}_{1}$

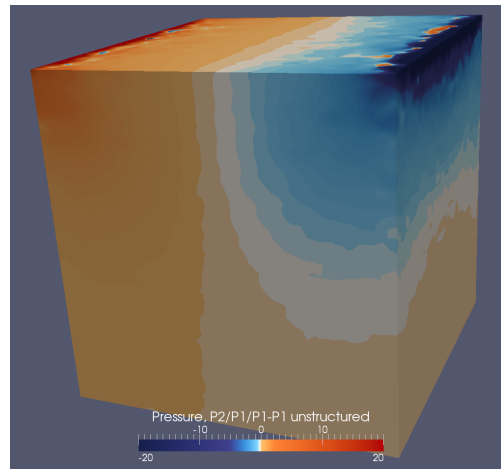

(c) $\left(\mathcal{P}_{2}, \mathcal{P}_{1}, \mathcal{P}_{1}\right)-\mathcal{P}_{1}$

Figure 18: Comparing the pressure in a $3 D$ unstructured mesh.

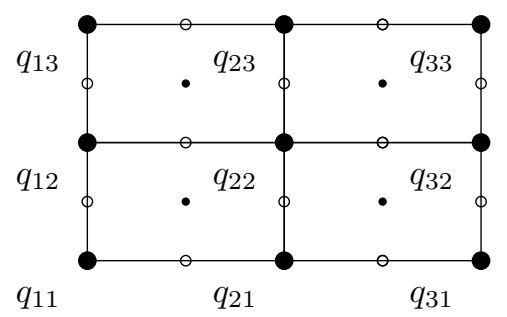

Figure 19: $\left(\mathcal{Q}_{2}, \mathcal{Q}_{1}\right)-\mathcal{Q}_{1}$ macroelement

loss of generality it can be assumed that the central vertex is located at the origin of coordinates; i.e. $q_{22}=(0,0)$ using the notation of Figure 19 .

Let us define the following $\mathrm{FE}$ spaces in $M$ :

$$
\begin{aligned}
U_{0, M} & =\left\{u_{h} \in H_{0}^{1}(M) \cap C^{0}(\bar{M}) /\left.u_{h}\right|_{K} \in \mathcal{Q}_{2}, \forall K \in M\right\}, \\
V_{0, M} & =\left\{v_{h} \in H_{0}^{1}(M) \cap C^{0}(\bar{M}) /\left.v\right|_{K} \in \mathcal{Q}_{1}, \forall K \in M\right\} \\
P_{M} & =\left\{p_{h} \in C^{0}(\bar{M}) /\left.p\right|_{K} \in \mathcal{Q}_{1}, \forall K \in M\right\}
\end{aligned}
$$

Let us consider the $\mathcal{Q}_{1}$ function $p_{h}$ defined as

$$
p_{h}(x, y)= \begin{cases}a+c y & \text { if } y>0 \\ a-c y & \text { if } y \leq 0\end{cases}
$$

where $a, c \in \mathbb{R}, c \neq 0$. Then each $v_{h} \in V_{0, M}$ can be written as $\alpha \phi_{22}$ for any $\alpha \in \mathbb{R}$, where $\phi_{22}$ is the $\mathcal{Q}_{1}$ basis function that is equal to 1 in $q_{22}$ and zero in all other vertices of $M$. Hence, if we apply the $2 \mathrm{D}$ trapezoidal rule (which is exact in $\mathcal{Q}_{1}$ ) and taking into account that $\partial_{x} p_{h}=0$,

$$
\begin{aligned}
\int_{M}\left(\partial_{x} u_{h}+\partial_{y} v_{h}\right) p_{h} & =-\int_{M} v_{h} \partial_{y} p_{h}=-\frac{c}{\left|M^{+}\right|} \int_{M^{+}} \alpha \phi_{22}+\frac{c}{\left|M^{-}\right|} \int_{M^{-}} \alpha \phi_{22} \\
& =\frac{c}{\left|M^{+}\right|} \sum_{K \subset M^{+}} \frac{|K|}{4} \alpha-\frac{c}{\left|M^{-}\right|} \sum_{K \subset M^{-}} \frac{|K|}{4} \alpha=\frac{c}{4} \alpha-\frac{c}{4} \alpha=0 .
\end{aligned}
$$

Therefore the combination $\left(\mathcal{Q}_{2}, \mathcal{Q}_{1}\right)-\mathcal{Q}_{1}$ is not stable in quadrangular macro-elements.

As in Section 4, it is not difficult to extend the previous counterexample to demonstrate that there exists a family of not null pressures $p_{h} \in P_{h}$ satisfying $\left(\nabla \cdot\left(u_{h}, v_{h}\right), p_{h}\right)=0$ for all $u_{h} \in U_{h}$ 


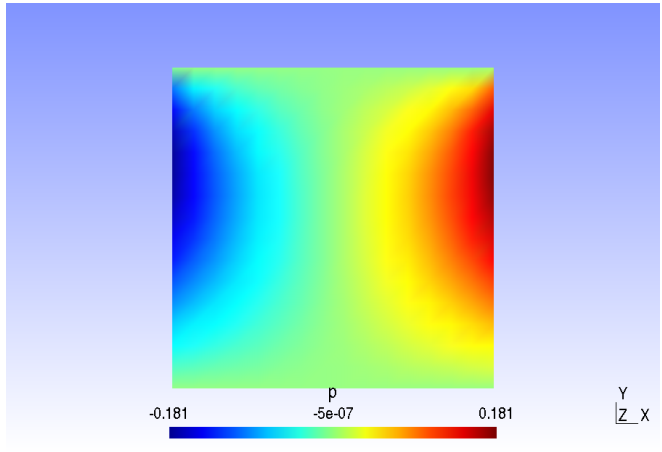

(a)

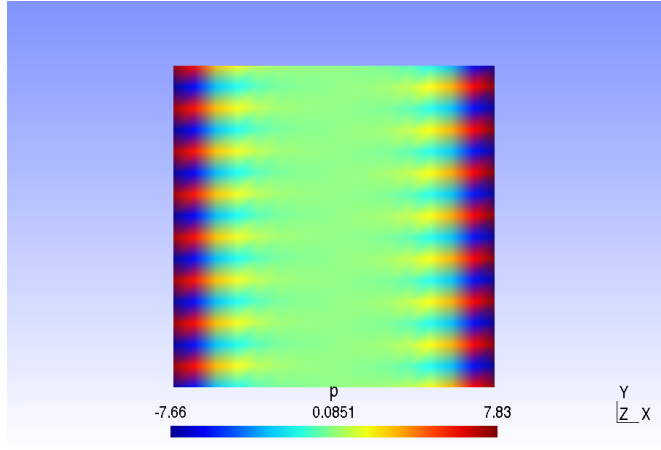

(b)

Figure 20: Pressure for $\left(\mathcal{Q}_{2}, \mathcal{Q}_{2}\right)-\mathcal{Q}_{1}$ (left) and $\left(\mathcal{Q}_{2}, \mathcal{Q}_{1}\right)-\mathcal{Q}_{1}$ (right). The latter, presents spurious ocillations.

and $v_{h} \in V_{h}$. Hence, in rectangular structured meshes, the Stokes inf-sup condition (6) does not hold for the $\left(\mathcal{Q}_{2}, \mathcal{Q}_{1}\right)-\mathcal{Q}_{1}$ combination.

Test $11\left(\left(\mathcal{Q}_{2}, \mathcal{Q}_{1}\right)-\mathcal{Q}_{1}\right.$ and cavity test). Numerical experiments confirm the $\left(\mathcal{Q}_{2}, \mathcal{Q}_{1}\right)-\mathcal{Q}_{1}$ instability, as can be seen in Figure 20. We consider the $2 D$ Stokes problem $(1)-(2)$ in $\Omega=(0,1)^{2}$. Dirichlet boundary conditions for $u$ have been applied; $u=1$ on the top $\{y=1\}$ and $u=0$ on the rest of $\partial \Omega$. About $v$, a natural boundary condition $\frac{\partial v}{\partial \mathbf{n}}=0$ has been chosen on $\partial \Omega$. Both stable $\left(\mathcal{Q}_{2}, \mathcal{Q}_{2}\right)-\mathcal{Q}_{1}$ and unstable $\left(\mathcal{Q}_{2}, \mathcal{Q}_{1}\right)-\mathcal{Q}_{1}$ combinations of $\mathrm{FE}$ have been tested in a mesh composed of $15 \times 15$ rectangular elements. As expected, Figure 20 shows spurious pressure oscillations in the latter case. This experiment has been programmed in C++ with LibMesh [KPSC06], a parallel FE library which supports natively the $\mathcal{Q}_{1}$ and $\mathcal{Q}_{2}$ elements.

\section{Conclusions}

In this paper we have developed two new families of FE spaces approximating the Stokes problem, where continuous and piecewise linear functions $\mathcal{P}_{1}$ are enriched, only for one component of the velocity, by either bubble $\mathcal{P}_{1, b}$ or quadratic polynomial $\mathcal{P}_{2}$. These combinations have been denoted, in the $2 D$ case, by $\left(\mathcal{P}_{1, b}, \mathcal{P}_{1}\right)-\mathcal{P}_{1}$ and $\left(\mathcal{P}_{2}, \mathcal{P}_{1}\right)-\mathcal{P}_{1}$.

In order to compare the use of bubble functions, we have seen that combination $\left(\mathcal{P}_{1, b}, \mathcal{P}_{1}\right)-\mathcal{P}_{1}$ in uniformly unstructured meshes is more efficient than the well-known mini-element $\left(\mathcal{P}_{1, b}, \mathcal{P}_{1, b}\right)-\mathcal{P}_{1}$, because $\left(\mathcal{P}_{1, b}, \mathcal{P}_{1}\right)-\mathcal{P}_{1}$ preserves the first order accurate of $\left(\mathcal{P}_{1, b}, \mathcal{P}_{1, b}\right)-\mathcal{P}_{1}$ while requires a minor number of degrees of freedom and then a smaller computational effort.

On the other hand, we have also proved that combination $\left(\mathcal{P}_{2}, \mathcal{P}_{1}\right)-\mathcal{P}_{1}$ in most uniformly unstructured meshes is stable but it does not preserve the second order accuracy of the well-known Taylor-Hood element $\left(\mathcal{P}_{2}, \mathcal{P}_{2}\right)-\mathcal{P}_{1}$, although $\left(\mathcal{P}_{2}, \mathcal{P}_{1}\right)-\mathcal{P}_{1}$ uses a minor number of degrees of freedom.

Moreover, the stability constraints for $\left(\mathcal{P}_{2}, \mathcal{P}_{1}\right)-\mathcal{P}_{1}$ are more restrictive than for $\left(\mathcal{P}_{1, b}, \mathcal{P}_{1}\right)-\mathcal{P}_{1}$, because they depend not only on the structure of the mesh but also depend on the algebraic condition (34). Hence, in principle, the number of meshes where $\left(\mathcal{P}_{2}, \mathcal{P}_{1}\right)-\mathcal{P}_{1}$ is stable is slightly less than in the $\left(\mathcal{P}_{1, b}, \mathcal{P}_{1}\right)-\mathcal{P}_{1}$ case.

Finally, the same type of results are also deduced in $3 D$ domains for the case of bubble functions. 


\section{References}

[AG01] P. Azérad and F. Guillén. Mathematical justification of the hydrostatic approximation in the primitive equations of geophysical fluid dynamics. Siam J. Math. Ana., 33(4):847-859, 2001.

[AR09] Douglas N. Arnold and Marie E. Rognes. Stability of Lagrange elements for the mixed Laplacian. Calcolo, 2009.

[Azé94] P. Azérad. Analyse et approximation du problème de Stokes dans un bassin peu profond. C. R. Acad. Sci. Paris Sér. I Math., 318(1):53-58, 1994.

[Azé96] P. Azérad. Analyse des quations de Navier-Stokes en bassin peu profond et de l'quation de transport. PhD thesis, Neuchtel, 1996.

[BBF13] Daniele Boffi, Franco Brezzi, and Michel Fortin. Mixed Finite Element Methods and Applications, volume 44 of Springer Series in Computational Mathematics. Springer Berlin Heidelberg, Berlin, Heidelberg, 2013.

[BF91] F. Brezzi and M. Fortin. Mixed and Hybrid Finite Element Methods. Springer-Verlag, New-York, 1991.

[BL92] O. Besson and M.R. Laydi. Some estimates for the anisotropic Navier-Stokes equations and for the hydrostatic approximation. Math. Mod. and Num. Anal, Vol. 26(7):855-865, 1992.

[CB09] B. Cushman-Roisin and J. M. Beckers. Introduction to Geophysical Fluid Dynamics - Physical and Numerical Aspects. Academic Press, 2009.

[GR86] V. Girault and P.-A. Raviart. Finite element methods for Navier-Stokes equations. Springer-Verlag, 1986.

[GR09] C. Geuzaine and J.-F. Remacle. Gmsh: A 3-D finite element mesh generator with built-in pre- and post-processing facilities. International Journal for Numerical Methods in Engineering, 79:1309 - 1331, 2009.

[GR14] F. Guillén-González and J.R. Rodríguez-Galván. Analysis of the hydrostatic stokes problem and finiteelement approximation in unstructured meshes. Numer. Math., 2014. Accepted, doi 10.1007/s00211-0140663-8.

[KPSC06] B.S. Kirk, J.W. Peterson, R.H. Stogner, and G.F. Carey. libmesh: a c++ library for parallel adaptive mesh refinement/coarsening simulations. Eng. with Comput., 22(3):237-254, December 2006.

$\left[\mathrm{LMW}^{+} 12\right]$ Anders Logg, Kent-Andre Mardal, Garth N. Wells, et al. Automated Solution of Differential Equations by the Finite Element Method. Springer, 2012.

[PHLHM] O. Pironneau, F. Hecht, A. Le Hyaric, and J. Morice. FreeFEM++, http://www.freefem.org/.

[QZ07] J. Qin and S. Zhang. Stability and approximability of the P1-P0 element for Stokes equations. International Journal for Numerical Methods in Fluids, 54:497-515, 2007.

[Ste84] R. Stenberg. Analysis of mixed finite element methods for the Stokes problem: A unified approach. Math Comput, 42(165):9-23, January 1984.

[Ste90] R. Stenberg. A technique for analysing finite elements methods for viscuous incompressible flow. International Journal for Numerical Methods in Fluids, 11:835-948, 1990. 